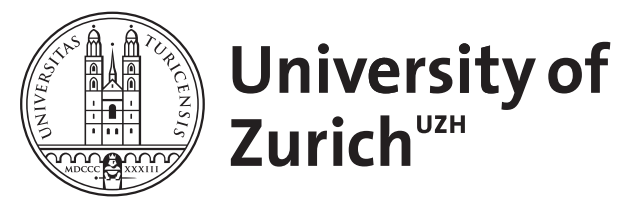

\title{
Shareholder Risk Measures
}

Rochet, Jean-Charles ; Coculescu, Delia

\begin{abstract}
The aim of this paper is to put forward a new family of risk measures that as the coherent/convex risk measures impose a preference order on random cash flows and can be interpreted as prices. But at the difference of the axiomatic approach of Artzner, Delbaen, Eber and Heath (1999) and the subsequent extensions of this model, our risk measures are associated with the optimal policies of shareholder value maximizing company. We study these optimal policies and the related risk measures that we call shareholder risk measures. We emphasize the fact that due to the specific corporate environment, in particular the limited shareholders' liability and the possibility to pay out dividends from the cash reserves, these risk measures are not convex. Also, they depend on the specific economic situation of the firm, in particular its current cash level, and thus they are not translation invariant. This paper bridges the gap between two important branches of mathematical finance: risk measures and optimal dividends.
\end{abstract}

DOI: https://doi.org/10.1111/mafi.12142

Posted at the Zurich Open Repository and Archive, University of Zurich

ZORA URL: https://doi.org/10.5167/uzh-149100

Journal Article

Accepted Version

Originally published at:

Rochet, Jean-Charles; Coculescu, Delia (2018). Shareholder Risk Measures. Mathematical Finance, 28(1):5-28.

DOI: https://doi.org/10.1111/mafi.12142 


\section{MATHEMATICAL An International Journal of Mathematics, FINANCE Statistics and Financial Economics}

\section{Shareholder Risk Measures}

\begin{tabular}{|r|l|}
\hline Journal: & Mathematical Finance \\
\hline Manuscript ID & MAFI-2015-0003.R1 \\
\hline Date Submitted by the Author: & 13-Oct-2015 \\
\hline Complete List of Authors: & $\begin{array}{l}\text { Coculescu, Delia; University Zurich, Banking and Finance } \\
\text { Rochet, Jean-Charles; University of Zurich, Department of Banking and } \\
\text { Finance }\end{array}$ \\
\hline Keywords: & $\begin{array}{l}\text { risk measures, non convex risk measures, cash reserves management, } \\
\text { dividend optimization }\end{array}$ \\
\hline
\end{tabular}

SCHOLARONE

Manuscripts 


\title{
SHAREHOLDER RISK MEASURES
}

\author{
DELIA COCULESCU AND JEAN-CHARLES ROCHET
}

\begin{abstract}
The aim of this paper is to put forward a new family of risk measures that -as the coherent/convex risk measures- impose a preference order on random cash flows and can be interpreted as prices. But at the difference of the axiomatic approach of Artzner, Delbaen, Eber and Heath (1999) and the subsequent extensions of this model, our risk measures are associated with the optimal policies of shareholder value maximizing company. We study these optimal policies and the related risk measures that we call shareholder risk measures. We emphasize the fact that due to the specific corporate environment, in particular the limited shareholders' liability and the possibility to pay out dividends from the cash reserves, these risk measures are not convex. Also, they depend on the specific economic situation of the firm, in particular its current cash level, and thus they are not translation invariant. This paper bridges the gap between two important branches of mathematical finance: risk measures and optimal dividends.
\end{abstract}

\section{INTRODUCTION}

The aim of this paper is to put forward a new family of risk measures that are associated with the optimal policies of shareholder value maximizing company. We call them shareholder risk measures. Due to the specific corporate environment, in particular the limited shareholders' liability and the possibility to pay out dividends from the cash reserves, these risk measures are not convex. Also, they depend on the specific economic situation of the firm, in particular the current cash level, and thus they are not translation invariant.

In the work by Artzner and al. [2], a risk measure $\rho$ is defined using a set of axioms, which represent some properties that a risk measure should have in an abstract set-up: $\rho$ is a real-valued function which is positively homogeneous, subadditive, translation invariant and monotone. Such risk measures are called coherent. An important extension of the concept of coherent risk measures is the notion of convex risk measures, developed simultaneously by Föllmer and Schied [10] and Fritelli and Rosazza-Gianin [12], who proposed to relax the properties of subadditivity and positive homogeneity of $\rho$ with the weaker property of convexity:

$$
\rho(\lambda X+(1-\lambda) Y) \leq \lambda \rho(X)+(1-\lambda) \rho(Y), \quad \forall \lambda \in[0,1] .
$$

Department of Banking and Finance, Universität Zürich, Plattenstrasse 32, Zürich 8092, SWITZERLAND.

E-mail addresses: delia.coculescu@bf.uzh.ch, jean-charles.rochet@bf.uzh.ch.

Date: 4 december 2014.

2000 Mathematics Subject Classification. 15A52.

Key words and phrases. Risk measures, shareholder's risk measure, cash reserves management, dividend optimization, non-convex risk measures.

The research leading to these results has received funding from the European Research Council under the European Community's sevenths Framework Programme (FP7/2007-2013) grant agreements and from NCCR Finrisk (project Banking and Regulation). 
A risk measure $\rho(\cdot)$ imposes a preference order on random variables, but more than this, it corresponds to a price, hence it is expressed in monetary units. This last point is specific to risk measures, as opposed to the previously developed theory of expected utility of von Neumann and Morgenstern [20]. Many links with expected utility theory exist. For instance, in the case of a specific class of risk measures called shortfall risk measures, introduced by Föllmer and Schied [10]: if we consider a convex increasing function (not identically constant) $\ell: \mathbb{R} \rightarrow \mathbb{R}$, and an interior point $x_{0}$ in the range of $\ell$, the following function is a coherent risk measure:

$$
\rho(X):=\inf \left\{m \in \mathbb{R} \mid \mathbf{E}\left(\ell(-m-X) \leq x_{0}\right\}\right.
$$

Since the function $u(x):=-\ell(-x)$ is increasing concave, it can be interpreted as an utility function, and if $x_{0}=u(0)$ we can interpret $\rho$ above as an indifference price for the cash flow $X$.

Our aim in this paper is to develop new risk measures, that have similar properties as the convex risk measures (i.e., they impose a preference order on random cash flows and can be interpreted as prices). But, at the difference with the axiomatic approach of Artzner and al. [2] and its subsequent extensions, our risk measures are derived from the dividend policy - cash optimization problem faced by a firm under uncertainty. We thus make a bridge between two different problems in corporate finance, namely the optimal dividend policy and the measurement of risks.

In the dividend policy literature, given the characteristics of the cash flow process of a firm, two fundamental questions are addressed: when should dividends be distributed, and how much of the available cash should be distributed. The answer to these questions is called a dividend strategy. The classical approach, initiated by de Finetti [8], is to maximize the the expected present value of all (future) dividends that will be distributed until the company is ruined (we shall call this quantity the shareholder value). A rich literature has investigated this important topic (see a selection in Subsection 3.2 below).

Assuming that the objective of the firm is to maximize shareholder value ${ }^{1}$, we examine in this paper the impact of a random shock $X$ on the firm's cash reserves on shareholder value and use it as a measure of the risk of the random shock $X$. The properties of the resulting risk measures are afterwards analyzed. This approach has the advantage that it displays some specific features of the risk measures, such as the non convexity or the absence of translation invariance. Even though model dependent, our approach helps to understand from where these a-typical and perhaps at first sight non-intuitive features of our proposed risk measures come from: we show that the particular corporate environment, such as limited liability for shareholders and the possibility to pay dividends from the available cash, lead to such specific risk measures. Of course, in practice shareholder value depends on many other variables than cash reserves, such as assets profitability, growth opportunities, macroeconomic context. We abstract from these exogenous variables for the sake of clarity.

The remaining of the paper is organized as follows: Section 2 summarizes important concepts and definitions, Section 3 introduces the shareholder value maximization problem and characterizes the value function and the optimal dividend strategies. In Section 4 we establish the main properties of the SRM as opposed to the convex risk measures. Section 5

\footnotetext{
${ }^{1}$ This is indeed the mandate given to the manager by the owners of the firm. We leave aside agency conflicts, i.e., what happens when the manager pursues its own objectives at the expense of shareholders.
} 
is an introduction to the dynamic SRM, i.e., when the random shock affects the cash reserves at some future date.

\section{Some USEFul DEFinitions}

The dividend policy - cash optimization problem which will be at the base of our discussion gives raise to a value function that is not concave everywhere. It is useful therefore to introduce from now the concept of shareholder value function as:

Definition 2.1. A shareholder value function is a real valued function $v$ that maps the level of the cash reserves of the firm to its shareholder value, is increasing, nonnegative and satisfies $v(x) \geq x$.

Let $\mathcal{X}$ be a set of random variables on the probability space $(\Omega, \mathcal{F}, \mathbf{P})$. A risk measure is a mapping $\rho$ from a $\mathcal{X}$ to the real line. Let us now review two common ways of measuring a risk $X$ and then propose a third method which will be the one we shall study in this paper. In all cases, we use a shareholder value function $v$, and we suppose that an agent (the firm in our case) has already an available cash flow $k$. In order to emphasize the links with the utility theory of von Neumann and Morgenstern we introduce the function:

$$
u_{k}(x):=v(k+x)-v(k) .
$$

(1) Indifference price:

$$
\rho_{k}(X):=\inf \left\{m \mid \mathbf{E}\left[u_{k}(X+m)\right] \geq 0\right\}
$$

This is a generalization of the classical approach of shortfall risk measures seen as the distance to some convex acceptable set of random variables. Indeed, we can define the following acceptance set $\mathcal{A}_{k}:=\left\{x \in \mathcal{X} \mid \mathbf{E}\left(\ell_{k}(-X)\right] \leq 0\right\}$, with $\ell_{k}(x)=-u_{k}(-x)$. Then $\rho_{k}(X)=\inf \left\{m \mid X+m \in \mathcal{A}_{k}\right\}$ as in [2], but here $\mathcal{A}_{k}$ is not convex when $v$ is not concave.

Also, let us note that, on the level sets where $v$ is strictly increasing and concave, $\rho$ can be seen as the indifference price from the expected utility theory, since it is the unique solution $m$ of the equation: $\mathbf{E}\left[u_{k}(m+X)\right]=u_{k}(0)=0$. But $\rho$ can also be (and is commonly) interpreted as a capital requirement.

(2) Certainty equivalent:

$$
C E_{k}(X):=-\inf \left\{m \mid \mathbf{E}\left[u_{k}(X)\right] \leq u_{k}(m)\right\} .
$$

This measure corresponds to the concept of the certainty equivalent for $X$, i.e., the sure amount for which an agent remains indifferent to a lottery $X$. Indeed, if we suppose again that $v$ is concave so that $u_{k}(x)=v(k+x)-v(k)$ can be interpreted as an utility function in a classical sense, then $C E_{k}(x)=u_{k}^{-1} \mathbf{E}\left[u_{k}(X)\right]$, where $u_{k}^{-1}$ is the left-continuous inverse of $u_{k}$.

We put forward a new class of risk measures:

(3) Impact price, or shareholder risk measure:

$$
S R M_{k}(X):=v(k)-\mathbf{E}[v(k+X)]=-\mathbf{E}\left[u_{k}(X)\right]
$$

This is a measure of the loss in shareholder value that a random cash flow produces, supposing shareholder value function is $v$. We shall endogenize the function $v$ for the shareholder value maximization. Note that $S R M$ is conceptually different from the 
measures presented above in (1) and (2). It is related to the utility theory of von Neumann and Morgenstern with the following notable difference that the "utility" function we use:

(a) is not a concave function;

(b) depends on the current cash level in the firm. This means that as the cash level of the firm evolves, the "utility" function changes. Since the cash evolution is random, the utility function that will apply at a future date is a random variable;

(c) can be extended to a dynamic set-up.

(d) represents a price, i.e., can be expressed in monetary units;

We can already remark that the fact that the cash invariance property fails, is a consequence of the fact that $S R M$ is not defined as a distance to some acceptable set, as is the case of the coherent (and convex) risk measures, the financial interpretation being that cash has sometimes more value inside the company than in the shareholder's pocket (or the opposite). The nonconvexity of $S R M$ is linked to the nonconcavity of the function $v$. As the set of acceptable risks $\mathcal{A}_{k}:=\{X \mid S R M(X) \leq 0\}$ is not convex, a risk measure in the spirit of [2] is also non convex. At fixed level of the cash $k$ in the firm, we shall nevertheless identify some classes of risks for which $S R M$ is convex or translation invariant (within a given range of the cash being added/subtracted).

It can seem at a first sight surprising to have risk measures that may penalize diversification. Quasi-concavity of risk measures seems to be a very natural feature, and $S R M$ do not satisfy this property in general. Let us emphasize again that here we do not take the point of view of regulators, nor of a manager of some given portfolio in a company. From the shareholders' perspective, it is not always preferable to diversify risks involving both some positive payments and, as a downside, the default of the company. If we exclude these risks, we obtain what we shall call the class of sustainable risks, i.e., all random payments that do not produce the default of the company. $S R M$ is convex for the sustainable risks..

Finally, let us point out that it is of course possible to construct a robust version of the SRM by using a set of scenarios (i.e., probabilities measures) $\mathcal{Q}$ as:

$$
S R M_{k}(X):=\max _{\mathbf{Q} \in \mathcal{Q}} \mathbf{E}_{\mathbf{Q}}\left[-u_{k}(X)\right],
$$

this being perfectly compatible with the model we describe below (including in the dynamic version of SRM).

\section{Shareholder VAlue maximization}

This section presents the problem of optimal cash management of a firm optimizing the flow of dividends payed to the shareholders. Our aim is to emphasize some properties of the value function. We suppose that in absence of dividend payments, the available cash reserve of a firm follows, as time goes on, a jump diffusion. At any time, as long as the cash reserve remains positive, the firm can decide to pay a part of the available cash as dividends. The objective is to determine the dividend scheme that maximizes the expected discounted flow of dividends till the bankruptcy time (which is the first time when the cash process is less than zero).

The mathematical formulation is as follows. We suppose that $\left(\Omega, \mathcal{F},\left(\mathcal{F}_{t}\right)_{t \geq 0}, \mathbf{P}\right)$ is a filtered probability space and $\left(W_{t}\right)$ a standard Brownian motion. Let $\tilde{N}$ be an $\left(\mathcal{F}_{t}\right)$ compensated 
Poisson random measure,

$$
\tilde{N}(t, A)=N(t, A)-\mathbf{E}[N(t, A)]=N(t, A)-\nu(A) t
$$

where for every Borel set $A, N(t, A)$ is a Poisson random measure measuring the number of jumps with amplitude in $A$, up to and including time $t$ and $\nu$ is the Lévy measure associated with $N$ (here assumed to be finite).

Starting from an initial state $k \in \mathbb{R}$ for the cash level and given a dividend policy $\left(\xi_{t}\right)$ (i.e., our control process), the dynamics of the cash reserve process of the firm is governed by:

$$
\begin{aligned}
& d C_{t}=b\left(C_{t}\right) d u+\sigma d W_{t}+\int_{\mathbb{R}} z \tilde{N}(d t, d z)-d \xi_{t}, \\
& C_{0^{-}}=k
\end{aligned}
$$

with $b(x)=\mu+r x$. The function $b$ corresponds to the sum of expected earnings and interests paid on the cash reserves $\left(C_{t}\right)$. The process $\left(\xi_{t}\right)$ is an adapted increasing process, right-continuous, with $\xi_{0^{-}}=0$, representing the cumulated dividend payments till time $t$ (our control process). Hence, the dynamics of the process in (3.1) represents the economically meaningful evolution of cash reserves. More general dynamics would make no sense in this context.

As it is common in the theory of Markov processes, we introduce a family of probability measures $\left(\mathbf{P}^{x}\right)_{x \in \mathbb{R}}$, corresponding to a translation of $\left(C_{t}\right)$ such that $C_{0-}=x$ under $\mathbf{P}^{x}$ and thus allow having arbitrary initial conditions. We write $\mathbf{P}^{k}=\mathbf{P}$. We denote $\mathbf{E}^{x}$ for the expectation operator under the measure $\mathbf{P}^{x}$.

Let:

$$
\tau=\inf \left\{t \geq 0 \mid C_{t}<0\right\}
$$

be the time of bankruptcy of the firm. Obviously $\left(C_{t}\right)$ and $\tau$ depend on the chosen dividend policy $\left(\xi_{t}\right)$. We use the simple notations that do not make explicit this dependence and believe that this does not create any confusions. Let

$$
J^{\xi}(x)=\mathbf{E}^{x}\left[\int_{0^{-}}^{\tau} e^{-\rho t} d \xi_{t}\right], \quad \rho>0 \text { constant. }
$$

be the expected total discounted amount of dividends payed to the shareholders up to the bankruptcy time. The notation $\int_{0^{-}}^{\tau}$ stands for $\int_{[0, \tau]}$. The discount rate $\rho$ is constant.

Moreover, the following conditions are assumed to hold:
(A1) $\mu>\int_{\mathbb{R}} z \nu(d z)$.
(A2) $\rho>r$.
(A3) $\sigma \neq 0$.

Let us point out that the case $\sigma=0$ is treated in Albrecher and Thonhauser [1].

We define the set of admissible controls $\mathcal{A}$ as the set of adapted, nondecreasing, càdlàg processes $\xi$ such that a unique strong solution $\left(C_{t}\right)$ of (3.1) exists, and such that for any stopping times $S \leq T$ we have: $\int_{S}^{T} C_{s} d \xi_{s} \geq 0$. This last condition ensures that no dividends are payed that would exceed the cash reserve available.

We assume that the objective of the firm is to implement such a dividend policy $\xi^{*}$ that maximizes the function $J^{\xi}$, that is:

$$
v(x):=\sup _{\xi \in \mathcal{A}} J_{5}^{\xi}(x)=J^{\xi^{*}}(x) .
$$


In the remaining of this section we aim to emphasize some properties of the function $v$ which are useful later on, in particular the fact that it is not a concave function on $\mathbb{R}$ but it is concave on $\mathbb{R}_{+}$.

Let us introduce the characteristic operator $\mathbb{L}_{C}$ defined on a function $F: \mathbb{R} \rightarrow \mathbb{R}$ as follows:

$$
\mathbb{L}_{C} F(x)=\lim _{U \downarrow x} \frac{\mathbf{E}^{x} F\left(C_{T_{U}}\right)-F(x)}{\mathbf{E}^{x} T_{U}}
$$

where the limit is taken over a family of open sets $U$ shrinking down to $x$ in $\mathbb{R}$ and $T_{U}=$ $\inf \left\{t \geq 0 \mid C_{t} \in U^{c}\right\}$. From the general theory of Markov processes we know that the characteristic operator $\mathbb{L}_{C}$ that corresponds the jump diffusion appearing in (3.1) when no dividends are payed (that is $\xi \equiv 0$ ) and applied to a $C^{2}(\mathbb{R})$ function $\phi$ takes the following integro-differential form:

$$
\mathbb{L}_{C} \phi(x)=b(x) \frac{\partial \phi}{\partial x}(x)+\frac{1}{2} \sigma^{2} \frac{\partial^{2} \phi}{\partial x^{2}}(x)+\int_{\mathbb{R}}\left(\phi(x+z)-\phi(x)-\frac{\partial \phi}{\partial x}(x) z\right) \nu(d z)
$$

Also, it will be useful to recall the following definition for the local time appearing in the solution of the Skorohod problem for jump diffusions:

Definition 3.1. Let $O \subset \mathbb{R}$ be an open set. The Skorohod problem for the reflected jump diffusion $C$ into $\bar{O}$ is to find a pair $\left(C_{t}, \xi_{t}\right)$ of càdlàg adapted processes such that the following conditions are satisfied:

$$
\begin{aligned}
& d C_{t}=b\left(C_{t}\right) d t+\sigma d W_{t}+\int_{\mathbb{R}} z \tilde{N}(d t, d z)-d \xi_{t}, \\
& C_{t} \in \bar{O}, \forall t \geq 0, \\
& \left(\xi_{t}\right) \text { has finite variation and } d \xi_{t}=0 \text { if } X_{t} \in O .
\end{aligned}
$$

The process $\xi$ is called the local time at the boundary $\partial O$ of the reflected process $C$.

3.1. A characterization of the optimal dividend strategy. The theorem below states some properties of the value function when it is sufficiently smooths and of the corresponding optimal dividend strategy.

Theorem 3.2. Suppose that there exists a function $\phi \in C^{2}((0, \infty)) \cap C(\mathbb{R})$ such that $\phi$ solves the following variational inequations:

$$
\begin{aligned}
\sup \left\{\mathbb{L}_{C} \phi(x)-\rho \phi(x), 1-\frac{\partial \phi}{\partial x}(x)\right\}=0, & \forall x \in(0, \infty) \\
\phi(x)=0, & \forall x \leq 0,
\end{aligned}
$$

with the set $\left\{x \in(0, \infty) \mid \mathbb{L}_{C} \phi(x)-\rho \phi(x)<0\right\}$ being non empty.

Then:

(i) There exists a constant $x^{*} \geq 0$ such that:

$$
O:=\left\{x \in(0, \infty) \mid \mathbb{L}_{C} \phi(x)-\rho \phi(x)=0 \text { and } \frac{\partial \phi}{\partial x}(x)>1\right\}=\left(0, x^{*}\right)
$$

(the non intervention set).

(ii) $\phi$ is concave on the interval $[0, \infty)$. 
(iii)

$$
v(x)=\phi(x) .
$$

(iv) The optimal control $\xi^{*}$ is the local time at $x^{*}$ of the jump diffusion $C$ reflected back into $\left(0, x^{*}\right]$.

Proof. In this proof, we se shall always use the notation $\phi^{\prime}(0)$ for the right-hand derivative at zero.

Let us introduce the following subset:

$$
D:=\left\{x \in(0, \infty) \mid \frac{\partial \phi}{\partial x}(x)=1 \text { and } \mathbb{L}_{C} \phi(x)-\rho \phi(x) \leq 0\right\}
$$

$O$ and $D$ are disjoint sets. Moreover, by definition of $\phi$ we have $O \cup D=(0, \infty)$. $O$ is open and the interior of $D$ is not empty (since the set $\left\{x \in(0, \infty) \mid \mathbb{L}_{C} \phi(x)-\rho \phi(x)<0\right\}$ is supposed non empty).

We now show that $O=\left(0, x^{*}\right)$ and hence $D=\left[x^{*}, \infty\right)$ for some $x^{*} \geq 0$ (with $x^{*}=0$ for $O=\emptyset)$.

If $\mu=b(0) \leq 0$ then $\phi(x)=x \vee 0$ is a solution of $(3.7),(3.8)$ and $D=(0, \infty)$, hence in this case (i) and (ii) hold.

We now study the case $\mu>0$. In this case, $0 \notin \bar{D}$ and hence $\exists \hat{x}>0$ such that $(0, \hat{x}) \in O$ and $\hat{x} \in D$ ( $D$ being non empty). Also, notice that in $D, \mathbb{L}_{C} \phi(x)-\rho \phi(x)=b(x)-\rho(x+K):=$ $h(x)$ with $K$ some constant such that $h(x) \leq 0$. The function $h(x)$ is strictly decreasing $\left(b^{\prime}(x)<\rho\right)$, hence if $y$ belongs to the interior of $D$ then $[y, \infty) \in D$. Hence $\exists x^{*}$ such that $\left[x^{*}, \infty\right) \in D$. In order to show that $D=\left[x^{*}, \infty\right)$, it remains to show that $D$ does not have isolated points, other configurations for $D$ being already excluded by the above remarks.

If $D$ has one ore several isolated points, then necessarily $\phi^{\prime}(x)$ is not monotonic, $D$ being precisely the set where $\phi^{\prime}$ attains its minimum. We will show that $D$ does not have isolated points by showing that $\phi^{\prime}(x)$ is necessarily monotonic on $(0, \infty)$. We shall see this simultaneously proves the concavity of $\phi$ on $[0, \infty)$.

Indeed, in $O, \phi$ satisfies:

$$
\frac{1}{2} \sigma^{2} \frac{\partial^{2} \phi}{\partial x^{2}}(x)=\rho \phi(x)-b(x) \frac{\partial \phi}{\partial x}(x)-\int_{\mathbb{R}}\left(\phi(x+z)-\phi(x)-\frac{\partial \phi}{\partial x}(x) z\right) \nu(d z):=g(x) .
$$

The function $g(x)$ satisfies $g(0)<0$, hence $\phi$ concave in $O$ near 0 . In other words, the function $\phi^{\prime}>1$ and decreasing in $O$ near 0 .

Suppose that $\phi^{\prime}$ is not monotonic in $\left(0, x^{*}\right]$, then there should be at least a local maximum of $\phi^{\prime}$ inside $\left(0, x^{*}\right)$, at a point say $x^{m}$ and a local minimum at a point say $x_{m}$ with $x_{m}<x^{m}$.

Our first task is to show that the global maximum of $\phi^{\prime}(x)$ is attained at $x=0$. Our second task is to prove (using this property of the global maximum) that $\phi^{\prime}(x)$ cannot have a local maximum at $x^{m}$.

For the moment, let us assume such a local maximum of $\phi^{\prime}$ at $x^{m} \in\left(0, m^{*}\right)$ exists and furthermore that it is a global maximum. Hence $\phi^{\prime \prime}(x)$ switches the sign at some point $x^{m} \in\left(0, x^{*}\right)$ from being positive to being negative and furthermore

$$
\phi^{\prime}\left(x^{m}\right)=\max _{\substack{x \in(0, \infty) \\ 7}} \phi^{\prime}(x)
$$


We now show that this is impossible. The sign of $\phi^{\prime \prime}(x)$ is given by the sign of the function $g(x)$, since by assumption (A3), $\sigma \neq 0$. We have that:

$$
g^{\prime}\left(x^{m}\right)=(\rho-r) \phi^{\prime}\left(x^{m}\right)+\left(\phi^{\prime}\left(x^{m}\right)-\int_{\left[-x^{m}, \infty\right)} \phi^{\prime}\left(x^{m}+z\right) \nu(d z)\right)>0
$$

(using: $\phi^{\prime \prime}\left(x^{m}\right)=0,(\mathrm{~A} 2)$ and the relation (3.9)). This shows that the relation (3.9) cannot hold, hence the maximum of $\phi^{\prime}(x)$ needs to be attained at the boundary, i.e., at $x=0$ and $\phi^{\prime}\left(x^{m}\right)$ can only be a local maximum. Now assume that the parameters of the problem enable this to happen, i.e., we have:

$$
g^{\prime}\left(x^{m}\right)<0
$$

Without loss of generality, we suppose $\phi^{\prime}\left(x^{m}\right)$ is the maximum value of $\phi^{\prime}$ that also satisfies $\phi^{\prime \prime}=0$ (i.e., $\phi^{\prime}\left(x^{m}\right)$ is the maximum among all local maximums that exist for $\phi^{\prime}$ inside the interval $\left.\left(0, x^{*}\right)\right)$.

As emphasized earlier, a local minimum needs to exist too, more precisely, $\exists 0<x_{m}<x^{m}$ such that $\phi^{\prime \prime}\left(x_{m}\right)=0$ and

$$
g^{\prime}\left(x_{m}\right)>0
$$

Without loss of generality, we assume that $x_{m}$ is the first local minimum of $\phi^{\prime}$, i.e., $\phi^{\prime}$ is decreasing on the interval $\left(0, x_{m}\right]$. Due to our assumptions on $x_{m}$ and $x^{m}$ we have that $A:=\left\{x \in(0, \infty) \mid \phi^{\prime}(x)>\phi\left(x^{m}\right)\right\} \subset\left[0, x_{m}\right)$ and $\phi^{\prime}$ decreasing in $A$. Since it is necessary to have $\phi^{\prime}\left(x^{m}\right)<\int_{\left[-x^{m}, \infty\right)} \phi^{\prime}\left(x^{m}+z\right) \nu(d z)$ (in order to make possible for (3.10) to hold), we have that $\int_{\left[-x^{m}, \infty\right)} \phi^{\prime}\left(x^{m}+z\right) \nu(d z)=\phi^{\prime}\left(y^{m}\right)$ for some $y^{m} \in A$. Since $x_{m}<x^{m}$ it follows that $\exists 0 \leq y_{m} \leq y^{m}<x_{m}$ such that $\int_{\left[-x_{m}, \infty\right)} \phi^{\prime}\left(x_{m}+z\right) \nu(d z)=\phi^{\prime}\left(y_{m}\right)$.

Substracting (3.11) from (3.10) and because $\phi^{\prime}$ decreasing on $\left[0, x_{m}\right]$ we obtain that:

$$
\begin{aligned}
(1+\rho-r)\left(\phi^{\prime}\left(x^{m}\right)-\phi^{\prime}\left(x_{m}\right)\right) & <\int_{[0, \infty)}\left(\phi^{\prime}\left(x^{m}+z\right)-\phi^{\prime}\left(x_{m}+z\right)\right) \nu(d z) \\
& =\phi^{\prime}\left(y^{m}\right)-\phi^{\prime}\left(y_{m}\right)<0 .
\end{aligned}
$$

But this is a contradiction, since obviously we must have $(1+\rho-r)\left(\phi^{\prime}\left(x^{m}\right)-\phi^{\prime}\left(x_{m}\right)\right)>0$ (both $\rho>r$ and $\phi^{\prime}\left(x^{m}\right)>\phi^{\prime}\left(x_{m}\right)$ must hold).

This proves that a local maximum for $\phi^{\prime}$ inside $\left(0, x^{*}\right)$ cannot exist, i.e. $\phi^{\prime \prime}$ cannot switch the sign from being positive to being negative in $\left(0, x^{*}\right)$. Hence, $\phi^{\prime}(x)$ is monotonically decreasing on $\left[0, x^{*}\right)$, i.e., $\phi$ is concave on $\left[0, x^{*}\right)$.

This proves also that $D=\left[x^{*}, \infty\right)$ and $O=\left(0, x^{*}\right)$ for some $x^{*} \geq 0$ (with $x^{*}=0$ for $O=\emptyset)$, hence $(\mathrm{i})$.

(ii) is a consequence of the fact that $\phi$ is $C^{2}$ concave on $\left(0, x^{*}\right)$ and linear on $\left(x^{*}, \infty\right)$.

The proof of (iii) is classical. Suppose that $x>0$. Choose any dividend policy $\xi \in \mathcal{A}$ and $t$ in the stochastic interval $[0, \tau]$. By Itô's formula for the semimartingale $C$ (recall that $\phi$ is 
assumed to be $\left.C^{2}(0, \infty)\right)$ we have:

$$
\begin{aligned}
e^{-\rho t} \phi\left(C_{t}\right)= & \phi(x)+\int_{0}^{t} e^{-\rho s}\left(\mathbb{L}_{C} \phi\left(C_{s}\right)-\rho \phi\left(C_{s}\right)\right) d s+\int_{0}^{t} e^{-\rho s} \sigma \frac{\partial \phi}{\partial x}\left(C_{s}\right) d W_{s} \\
& -\int_{0}^{t} e^{-\rho s} \frac{\partial \phi}{\partial x}\left(C_{s-}\right) d \xi_{t}^{c}+\sum_{0<t_{n}<t} e^{-\rho t_{n}} \Delta_{\xi} \phi\left(C_{t_{n}}\right)
\end{aligned}
$$

where $\left(t_{n}\right)$ are the jumping times of $\xi$ and $\left.\Delta_{\xi} \phi\left(C_{t_{n}}\right)=\phi\left(C_{t_{n}}\right)-\phi\left(C_{t_{n}}+\Delta \xi_{t_{n}}\right)\right)$ is the variation of $\phi$ due to the jump $\Delta \xi_{t_{n}}=\xi_{t_{n}}-\xi_{t_{n}-}$. Since $\phi$ is increasing and $\xi$ increasing, all $\Delta_{\xi} \phi\left(C_{t_{n}}\right) \leq 0$.

Therefore, using (3.7):

$$
0 \leq e^{-\rho t} \phi\left(C_{t}\right) \leq \phi(x)+\int_{0}^{t} e^{-\rho s} \sigma \frac{\partial \phi}{\partial x}\left(C_{s}\right) d W_{s} .
$$

The process $m_{t}:=\int_{0}^{t} e^{-\rho s} \sigma \frac{\partial \phi}{\partial x}\left(C_{s}\right) d W_{s}, t \geq 0$ is a local martingale. We take a localizing sequence $\left(T_{a}\right)_{a \geq 1}$ of this local martingale. We then define the increasing sequence of stopping times $\tau_{a}:=T_{a} \wedge \tau, a \geq 1$. Since the sequence $\left(m_{\tau_{a}}\right)$ is uniformly integrable, it follows from the above inequalities that also the sequence $\left(e^{-\rho \tau_{a}} \phi\left(C_{\tau_{a}}\right)\right)$ is uniformly integrable.

Taking expected value in (3.12) with $t=\tau_{a}$, we obtain:

$$
\begin{aligned}
\mathbf{E}^{x}\left[e^{-\rho \tau_{a}} \phi\left(C_{\tau_{a}}\right)\right]= & \phi(x)+\mathbf{E}^{x}\left[\int_{0}^{\tau_{a}} e^{-\rho t}\left(\mathbb{L}_{C} \phi\left(C_{t}\right)-\rho \phi\left(C_{t}\right)\right) d t-\int_{0}^{\tau_{a}} e^{-\rho t} \frac{\partial \phi}{\partial x}\left(C_{t-}\right) d \xi_{t}^{c}\right. \\
& \left.+\sum_{0<t_{n}<\tau_{a}} e^{-\rho t_{n}} \Delta_{\xi} \phi\left(C_{t_{n}}\right)\right] .
\end{aligned}
$$

From the inequations $(3.7)$ we have that $\mathbb{L}_{C} \phi(x)-\rho \phi(x) \leq 0$. It follows that:

$$
\phi(x) \geq \mathbf{E}^{x}\left[e^{-\rho \tau_{a}} \phi\left(C_{\tau_{a}}\right)\right]+\mathbf{E}^{x}\left[\int_{0}^{\tau_{a}} e^{-\rho t} \frac{\partial \phi}{\partial x}\left(C_{t-}\right) d \xi_{t}^{c}-\sum_{0<t_{n}<\tau_{a}} e^{-\rho t_{n}} \Delta_{\xi} \phi\left(C_{t_{n}}\right)\right]
$$

By the mean value theorem we have

$$
\Delta_{\xi} \phi\left(C_{t_{n}}\right)=-\frac{\partial \phi}{\partial x}\left(x_{n}\right) \Delta \xi_{t_{n}}
$$

for some point $x_{n}$ on the straight line between $C_{t_{n}}-\Delta \xi_{t_{n}}$ and $C_{t_{n}}$. Using the inequality $\frac{\partial \phi}{\partial x} \geq 1$, we obtain:

$$
\phi(x) \geq \mathbf{E}^{x}\left[e^{-\rho \tau_{a}} \phi\left(C_{\tau_{a}}\right)\right]+\mathbf{E}^{x}\left[\int_{0}^{\tau_{a}} e^{-\rho t} d \xi_{t}\right] .
$$

Letting $a \rightarrow \infty$, since $\left(\phi\left(C_{\tau_{a}}\right)\right)$ is uniformly integrable, $\lim _{a \rightarrow \infty} \tau_{a}=\tau$ and $\phi\left(C_{\tau}\right)=0$ a.s., we obtain that:

$$
\phi(x) \geq J^{\xi}(x)
$$

As this holds for any admissible control $\xi$, and since for $x \leq 0$ we have by definition $\phi(x)=$ $v(x)=0$, we conclude that:

$$
\phi(x) \geq v(x), \quad \forall x \in \mathbb{R}
$$


In particular, since it is admissible to pay immediately time 0 dividends in amount $C_{0}$, $v(x) \geq x$ and hence we must also have:

$$
\phi(x) \geq x .
$$

Now suppose that $\xi_{t}=L_{t}^{y}$, the local time at $y>0$ of the process $C$ reflected back into $(0, y]$, for some $y \geq 0$. Note that $L^{y} \in \mathcal{A}$. Indeed, $L^{y}$ is adapted, increasing, and càdlàg. Moreover, a unique strong solution of (3.1) when $\xi_{t}=L_{t}^{y}$ exists (see [7]).

Our aim is to show that there exists $x^{*} \geq 0$ such that the function $\phi(x):=\mathbf{E}^{x}\left[\int_{0}^{\tau} e^{-\rho t} d L_{t}^{x^{*}}\right]$ solves the problem (3.7)-(3.8) for every $x \in \mathbb{R}$. Combined with the inequality (3.13) this will prove that $L^{x^{*}}$ is the optimal strategy and $\phi=v$.

For the moment take any $y>0$ and denote $g^{y}(x):=\mathbf{E}^{x}\left[\int_{0}^{\tau} e^{-\rho t} d L_{t}^{y}\right]$. Given $x \in(0, y)$, choose $\epsilon$ such that $(x-\epsilon, x+\epsilon) \in(0, y)$ and denote $T_{\epsilon}:=\inf \left\{t \geq 0 \mid C_{t} \in(x-\epsilon, x+\epsilon)^{c}\right\}$. By the strong Markov property we have:

$$
\mathbf{E}^{x} g^{y}\left(C_{T_{\epsilon}}\right)=\mathbf{E}^{x} \mathbf{E}^{C_{T_{\epsilon}}} \int_{0}^{\tau} e^{-\rho t} d L_{t}^{y}=\mathbf{E}^{x} e^{\rho T_{\epsilon}} \int_{T_{\epsilon}}^{\tau} e^{-\rho t} d L_{t}^{y}=\mathbf{E}^{x} e^{\rho T_{\epsilon}} \int_{0}^{\tau} e^{-\rho t} d L_{t}^{y}
$$

We have used the fact that $T_{\epsilon} \leq \tau$ a.s. and $\int_{0}^{T_{\epsilon}} e^{-\rho t} d L_{t}^{y}=0$. Hence, we see that:

$$
\begin{aligned}
\mathbb{L}_{C} g^{y}(x) & =\lim _{\epsilon \downarrow 0} \frac{\mathbf{E}^{x} g^{y}\left(C_{T_{\epsilon}}\right)-g^{y}(x)}{\mathbf{E}^{x} T_{\epsilon}}=\lim _{\epsilon \downarrow 0} \frac{\mathbf{E}^{x}\left(e^{\rho T_{\epsilon}}-1\right) \int_{0}^{\tau} e^{-\rho t} d L_{t}^{y}}{\mathbf{E}^{x} T_{\epsilon}} \\
& =\lim _{\epsilon \downarrow 0} \frac{\mathbf{E}^{x}\left(e^{\rho T_{\epsilon}}-1\right) \mathbf{E}^{x}\left(\int_{0}^{\tau} e^{-\rho t} d L_{t}^{y}\right)}{\mathbf{E}^{x} T_{\epsilon}}=\rho g^{y}(x) .
\end{aligned}
$$

This shows that $\mathbb{L}_{C} g^{y}(x)-\rho g^{y}(x)=0$ for $x \in(0, y)$. Also, by definition of $L^{y}$ we have that $g^{y}(y+c)=g^{y}(y)+c, \forall c>0$. Therefore, for any $x \in(y, \infty) g^{y}(x)$ is linear (hence $\left.C^{2}\right)$ and it can be checked that both $\frac{\partial g^{y}}{\partial x}(x)=1$ and $\mathbb{L}_{C} g^{y}(x)-\rho g^{y}(x)<0$ hold.

Let us notice that $g^{y}(x)=0$ for $x \leq 0$, that is equation (3.8) is verified by $g^{y}$.

In order to prove points (iii) and (iv) of the theorem it is now sufficient to show that $\exists x^{*}$ in $[0, \infty)$ such that $g^{x^{*}}$ is $C^{2}((0, \infty))$. But this fact is ensured by the fact that a $C^{2}$ solution to (3.7) is assumed to exist plus the previous remark that the set $O$ must be of the form $\left(0, x^{*}\right)$.

The next Corollary emphasizes the fact that the function $v$ is a shareholder value function (see Definition 2.1) and therefore the corresponding risk measures will not be convex. As we have shown, $v$ is concave on $(0, \infty)$, the concavity breaks down on $[-\infty, \infty]$ as a consequence of the existence of the limited liability for shareholders.

Corollary 3.3. Under the assumptions of Theorem 3.2, the value function $v(x)$ is a shareholder value function. It is constant on $\mathbb{R}_{-}$and concave on $\mathbb{R}_{+}$.

3.2. Some bibliographical comments and particular examples. There exists an extensive literature on barrier strategies for dividends, i.e., dividend payouts that are the local time of the cash process at an upper level. Let us mention that the book by Schmidli [23], treats among other topics the problem of dividend distributions in insurance companies and the reader can find an overview of the topic.

These models were initially developed by actuaries, where the cash process was considered to be the surplus of an insurance company. Dividends distributions of barrier type have 
been shown to be optimal for some types of cash processes. The first model of this type was elaborated by de Finetti [8]; he showed that if the risk/surplus process evolves as a random walk with step sizes \pm 1 , then an optimal way of paying out dividends is according to a barrier strategy. In the case of continuous-time models, the problem of finding the optimal dividend strategy has been studied extensively in the Cramér-Lundberg setting (Gerber [13]; Azcue and Muler [5]) and in the diffusion setting (Jeanblanc and Shiryaev [15]; Asmussen and Taksar [3]; Gerber and Shiu [14]). It was recently generalized to the spectrally negative Lévy model by Avram et al. [4], Kyprianou and Palmowski [16] and Loeffen [19], where the surplus is a general Lévy process with only negative jumps.

In the Cramér-Lundberg setting, the cash process is described by a Lévy process without the Brownian part (this would correspond to our model with $r=\sigma=0$ ). Our condition (A3) was important in our proof of the Theorem 3.2 and it makes that our model does not to fit the Cramér-Lundberg setting. Notice that Gerber [13] has shown that not only barrier but also band strategies can be optimal in the Cramér-Lundberg setting. A band strategy is such that the cash process is reflected at an upper barrier and absorbed to zero by paying a dividend once it crosses a lower barrier. This strategy can be optimal for some spectrally negative Lévy cash process (see also Azcue and Muler [5]) and for some models falling in the class studied in Abrecher and Thonhauser [1] (here again not containing a Brownian part).

We should emphasize that in the case of band strategies, the value function $v(x)$ is non concave even on $\mathbb{R}_{+}$, and also non smooth (in the sense that the first derivative is discontinuous). Concrete examples can be found for instance in Azcue and Muler [5] and Abrecher and Thonhauser [1]. In this case the value function remains however a shareholder value function (in the sense of our Definition 2.1) and a supremum of concave functions.

In the case of our model, the brownian component makes that paying out the cash entirely as a dividend triggers the default of the firm. Hence, we have the boundary condition $v(0)=0$. This property excludes to have band strategies, as optimal strategies, even in the situations where the value function would not be smooth (i.e., $C^{2}(0, \infty)$ ).

Below we give some examples of cash processes and the associated value functions.

3.2.1. Brownian Motion. This model is presented in Jeanblanc-Picqué and Shiryaev [15] and Shreve, Lehoczky and Gaver [24]. Suppose $b(x) \equiv \mu>0$ and $N(t, A) \equiv 0$ for any Borel set $A$. In this case we obtain:

$$
v(x):=\frac{e^{r_{1} x}-e^{r_{2} x}}{r_{1} e^{r_{1} x^{*}}-r_{2} e^{r_{2} x^{*}}} \quad 0 \leq x \leq x^{*}
$$

where $x^{*}=\frac{1}{r_{1}-r_{2}} \log \frac{r_{2}^{2}}{r_{1}^{2}}$ and $r_{1}>0>r_{2}$ are the roots of the equation: $\sigma^{2} r^{2}+2 \mu r-2 \rho=0$. $v(x)=v\left(x^{*}\right)+x-x^{*}$ for $x>x^{*}$.

3.2.2. Linear drift. Suppose that $b(x)=\mu+r x$ and $N(t, A) \equiv 0$ for any Borel set $A$.

We obtain that $v$ is solution of:

$$
\begin{aligned}
\frac{\sigma^{2}}{2} v^{\prime \prime}(x)+(\mu+r x) v^{\prime}(x)-\rho v(x) & =0 \\
v(0) & =0 \\
v^{\prime}\left(x^{*}\right) & =1
\end{aligned}
$$

11 


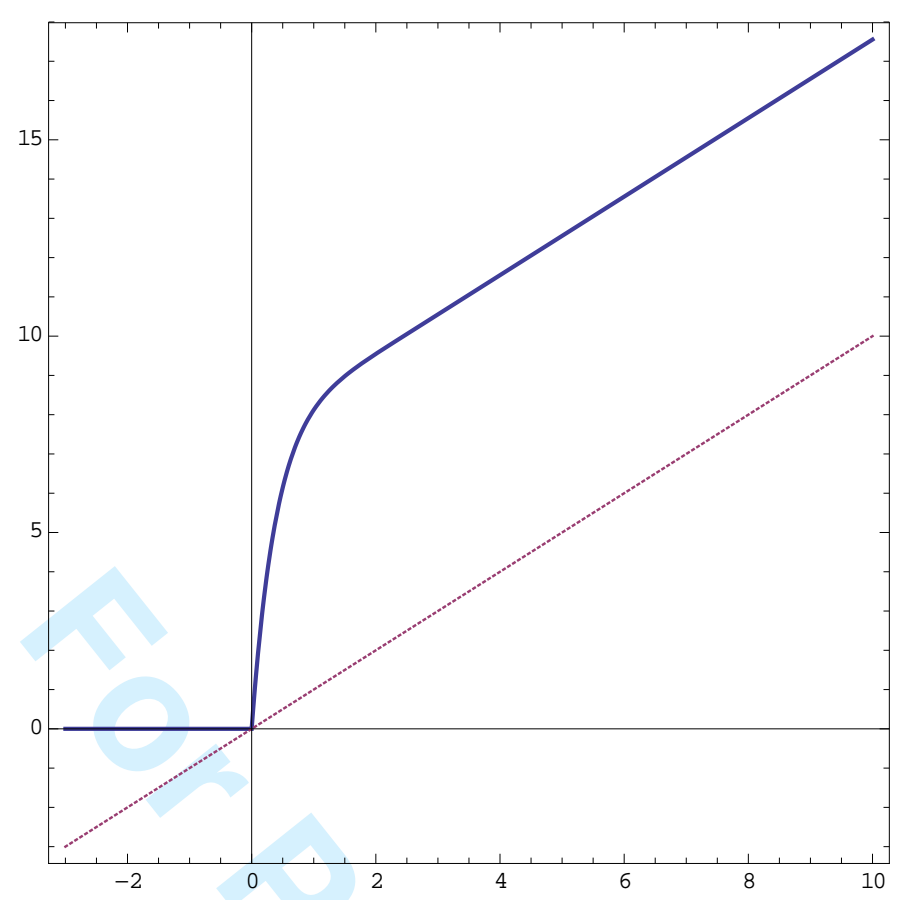

Figure 1. Value function $v(x)$ when the cash process $X$ is a Brownian Motion.

A change of variables: $f(y):=v(x) \exp \left(\frac{\sigma^{2}}{2}\left(r \frac{x^{2}}{2}+\mu x\right)\right)$, with $y=\sigma^{2}(\mu+r x)^{2} / 4 r$, leads to differential equation:

$$
y f^{\prime \prime}+\left(\frac{1}{2}-y\right) f^{\prime}-\lambda f=0
$$

with $\lambda:=\left(r-\rho \sigma^{2} / 2\right) / 2 r$.

We denote by $F(a, b, x)$ the standard confluent hypergeometric function (or Kummer's function) which arises as a solution of confluent hypergeometric differential equation $x y^{\prime \prime}+$ $(b-x) y^{\prime}-a y=0$. The solution of the equation (3.17) is:

$$
f(y)=C_{1} f_{1}(y)+C_{2} f_{2}(y)
$$

where $C_{1}$ et $C_{2}$ are constants and $f_{1}$ and $f_{2}$ are two independent solutions:

$$
\begin{aligned}
& f_{1}(y)=F(\lambda, 1 / 2, y) \\
& f_{2}(y)=y^{1 / 2} F(\lambda+1 / 2,3 / 2, y) .
\end{aligned}
$$

We obtain:

$$
v(x)=e^{\frac{\sigma^{2}}{2}\left(r \frac{x^{2}}{2}+\mu x\right)}\left(C_{1} F\left(\lambda, \frac{1}{2}, \frac{\sigma^{2}(\mu+r x)^{2}}{4 r}\right)+C_{2} \frac{\sigma(\mu+r x)}{2 \sqrt{r}} F\left(\lambda+\frac{1}{2}, \frac{3}{2}, \frac{\sigma^{2}(\mu+r x)^{2}}{4 r}\right)\right)
$$

(with the constants $C_{1}$ and $C_{2}$ fully determined by the boundary conditions).

3.2.3. Negative, exponentially distributed jumps. The case with $r=0, \nu(d z)=\mathbf{1}_{z<0} \lambda \delta e^{-\delta z} d z$ is studied in Belhaj [6] (see page 319 for the optimal value function and the barrier level in this model). 


\section{Properties of Shareholder Risk MeAsures}

We want to establish some properties of the shareholder risk measure $(S R M)$ generated by the shareholder value function $v$ that is the solution of the optimization problem from the previous section, or more generally using a shareholder value function that is concave on $(0, \infty)$ and is linear and on $\left(x^{*}, \infty\right)$ with $x^{*} \geq 0$ (one can have in mind Figure 1 ). The objective is too see how different the SRM is as compared to the classical convex risk measures.

We recall that a convex risk measure $\rho: \mathcal{X} \rightarrow \mathbb{R}$ is defined by the following properties:

(1) Monotonicity: If $X \leq Y$, then $\rho(X) \geq \rho(Y)$.

(2) Translation Invariance: If $m \in \mathbb{R}$, then $\rho(Y+m)=\rho(Y)-m$.

(3) Convexity: For any $\lambda \in \mathbb{R}, \rho(\lambda X+(1-\lambda) Y) \leq \lambda \rho(X)-(1-\lambda) \rho(Y)$.

4.1. Shareholder risk measures: a definition and some properties. Assume that we are time zero and an amount $k \in\left[0, x^{*}\right]$ of cash is available in the firm. Then, as described in the previous section, the shareholders value their shares as $v(k)$. If a random cash flow $X$ is about to affect the cash available in the firm, then the shareholders measure the risk of this random cash flow by its impact on their shareholder value.

We therefore define the following:

Definition 4.1. (1) We denote by $\mathcal{X}$ the class of random variables $X$ on $(\Omega, \mathcal{F})$ such that $\mathbf{E}[v(k+X)]<\infty$. The (instantaneous) shareholder risk measure for any $X \in \mathcal{X}$ is defined as:

$$
\operatorname{SRM}(X):=v(k)-\mathbf{E}[v(k+X)]
$$

(2) We denote $\mathcal{X}_{k}:=\{X \in \mathcal{X} \mid X>-k\}$ i.e., the set of risks bounded below by $-k$. $\mathcal{X}_{k}$ is called the set of sustainable risks. Each risk in this class does not trigger the firm's default, since the firm has sufficient cash to absorb its possible losses.

(3) The set of random variables $X$ satisfying $\mathbf{E}[v(k+X)] \geq v(k)$ or equivalently $S R M(X) \leq$ 0 is called the set of acceptable risks.

The intuition behind the definitions above is simple. If the cash process of the firm is impacted by a random variable $X$, then the value of the cash process changes from $k$ to $k+X$ and the value function from $v(k)$ to $v(k+X)$. The SRM evaluates the impact on the value function just before the random shock arrives, i.e., when there is no time to adjust the dividend policy before the occurrence of the shock. If the risk $X$ is such that $\mathbf{E}[v(k+X)]<v(k)$ it would be more convenient for shareholders to refuse being exposed to the risk $X$. We say that such risks are not acceptable. Note that concept of acceptability here is different in its interpretation from the one introduced in Delbaen and al. [2], where a risk is not acceptable when it requires additional capital. In our setting, shareholders take only the decision of the dividend policy and we do not allow for new capital injections.

Notation 4.2. With any random variable $X \in \mathcal{X}$ we attach a random variable $X_{k} \in \mathcal{X}_{k}$ by:

$$
X_{k}:=X \mathbf{1}_{\{X>-k\}}-k \mathbf{1}_{\{X \leq-k\}}=\max \{X,-k\} .
$$

Proposition 4.3 (Basic properties of the shareholder risk measure). SRM satisfies the following properties:

(i) $\operatorname{SRM}(0)=0$. More generally, if $X$ is such that $X_{k}=0$ a.s., then $\operatorname{SRM}(X)=0$.

(ii) General upper bound: $\operatorname{SRM}(X) \leq v(k)$. 
(iii) Monotonicity: if $X$ and $Y$ are such that $X_{k}>Y_{k}$ a.s. then $S R M(X)<S R M(Y)$.

(iv) For $X \geq x^{*}-k$ and $m \geq x^{*}-(k+X)$ SRM has the property of translation invariance:

$$
\operatorname{SRM}(X+m)=\operatorname{SRM}(X)-m \text {. }
$$

(v) Quasi-convexity and quasi-concavity (partial): if $X$ and $Y$ are such that $X_{k} \geq Y_{k}$ a.s., then for $\lambda \in(0,1)$,

$$
\min \{S R M(X), S R M(Y)\} \leq S R M(\lambda X+(1-\lambda) Y) \leq \max \{S R M(X), S R M(Y)\} .
$$

(vi) Law equivariance: if $X$ and $Y$ are such that $X_{k}$ and $Y_{k}$ have the same distribution, then $\operatorname{SRM}(X)=\operatorname{SRM}(Y)$.

Proof. (iii) Since $X_{k}>Y_{k}$ a.s. and $v$ is strictly increasing on $[0, \infty)$, we have that $v(k+$ $X)=v\left(k+X_{k}\right)>v\left(k+Y_{k}\right)=v(k+Y)$ and the result follows from the definition of SRM.

(iv) consequence of the fact that $v(x)$ is linear for $x>x^{*}$.

(v) We suppose that $X$ and $Y$ are such that $X_{k} \geq Y_{k}$ a.s.. In this case $v(k+X) \geq v(k+Y)$ a.s. and therefore:

$$
\begin{aligned}
\mathbf{E}[v(k+\lambda X+(1-\lambda) Y)] & \geq \mathbf{E}[\min (v(k+X), v(k+Y))] \\
& =\mathbf{E}[v(k+Y))]=\min \{\mathbf{E}[v(k+X)], \mathbf{E}[v(k+Y)]\}
\end{aligned}
$$

and:

$$
\begin{aligned}
\mathbf{E}[v(k+\lambda X+(1-\lambda) Y)] & \leq \mathbf{E}[\max (v(k+X), v(k+Y))] \\
& =\mathbf{E}[v(k+X))]=\max \{\mathbf{E}[v(k+X)], \mathbf{E}[v(k+Y)]\}
\end{aligned}
$$

It follows that the properties quasi-concavity and the quasi-convexity of SRM are satisfied for $X$ and $Y$ when $X_{k}>Y_{k}$ a.s..

We now write an equivalent form for the acceptable $S R M$, which emphasizes some similarities and differences with a class of law-equivariant or law-determined risk measures (also known as law-invariant risk measures). Kusuoka [18] has shown that every law equivariant, commonotonically additive risk measure can be represented as the expectation of the risk under a convexly distorted distribution. Namely, there exists a distortion $D$ (i.e., $D:[0,1] \rightarrow[0,1]$ increasing, right continuous, with $D(0)=0$ and $D(1)=1$ ) which is convex such that:

$$
\rho(X)=-\int_{0}^{1} F_{X}^{-1}(u) d D(u)=-\int_{\mathbb{R}} x d D \circ F_{X}(x),
$$

where $F_{X}(x):=\mathbf{P}(X \leq x)$.

Notation 4.4. On the interval $\left[0, x^{*}\right]$, the function $v$ is strictly concave therefore $v^{\prime}$ is invertible. We denote by $\left(v^{\prime}\right)^{-1}$ the inverse of $v^{\prime}$. By the mean value theorem, we have that for $x>-k$, there exists a point $y$ between $k$ and $x+k$ such that $v(k+x)-v(k)=x v^{\prime}(y)$. Since we have $k \in\left(0, x^{*}\right)$, the point $y$ also satisfies $y \in\left(0, x^{*}\right)$. Therefore, we define the function:

$$
g_{k}(x)=: \begin{cases}k & \text { if } x=0, \\ \left(v^{\prime}\right)^{-1}\left(\frac{v(k+x)-v(k)}{x}\right) & \text { if } x \in \mathbb{R}^{*}\end{cases}
$$


We also introduce the notations:

$$
\begin{gathered}
F_{X}(x):=\mathbf{P}(X \leq x) \\
F_{X_{k}}(x):=\mathbf{P}\left(X_{k} \leq x\right)
\end{gathered}
$$

Proposition 4.5 (Representation of the shareholder risk measure).

$$
S R M(X)=-\int_{\mathbb{R}} x v^{\prime}\left(g_{k}(x)\right) d F_{X_{k}}(x)
$$

Therefore:

(i) If $X>0$ a.s., we have that $\int_{\mathbb{R}} x v^{\prime}\left(g_{k}(x)\right) d F_{X_{k}}(x) \geq \mathbf{E}\left(X_{k}\right)=\mathbf{E}[X]$ hence:

$$
\operatorname{SRM}(X) \leq-\mathbf{E}(X)
$$

(ii) If $X<0$ a.s., we have that $\int_{\mathbb{R}} x v^{\prime}\left(g_{k}(x)\right) d F_{X_{k}}(x) \leq \mathbf{E}\left[X_{k}\right]$ hence:

$$
\operatorname{SRM}(X) \geq-\mathbf{E}\left(X_{k}\right)
$$

Let us comment on the above representations of $\rho$ versus $S R M$. Any law invariant commonotone risk measure for $X$ can be written as an expected value of $-X$ under a new probability measure. This probability measure is obtained by distorting the law of $X$ using a given function $D$ (thus for risks having different laws we get different probabilities after distortion). When computing the SRM for $X$, we also compute the expectation of $-X$, but under a measure which is not a probability measure anymore. More precisely, because $v^{\prime}(x) \geq 1$ on $\mathbb{R}_{+}$we have that the new measure has a total mass which exceeds 1 . The new measure depends not only on the law of $X$ (in fact on the law of $X_{k}$ ), but also on the level of cash in the firm $k$. The higher the cash level, the lower the total mass of our measure change.

\subsection{Further properties of SRM: the case of bounded risks.}

Proposition 4.6 (Convexity for sustainable risks). Let $X \in \mathcal{X}_{k}$ and $Y \in \mathcal{X}_{k}$. Then, for any $\lambda \in[0,1]$ we have:

$$
\operatorname{SRM}(\lambda X+(1-\lambda) Y) \leq \lambda S R M(X)-(1-\lambda) S R M(Y) .
$$

The same holds, if, more generally, $\{X<-k\}=\{Y<-k\}$ (thus $X$ and $Y$ need not be sustainable).

Proof. $\mathcal{X}_{k}$ is convex and $v$ is concave on $\mathbb{R}_{+}$. Hence for $X \in \mathcal{X}_{k}$ and $Y \in \mathcal{X}_{k}$ :

$$
\begin{aligned}
S R M(\lambda X+(1-\lambda) Y) & =v(k)-\mathbf{E}[v(\lambda(k+X)+(1-\lambda)(k+Y))] \\
& \leq v(k)-\mathbf{E}[\lambda v(k+X)+(1-\lambda) v(k+Y)] \\
& =\lambda S R M(X)-(1-\lambda) S R M(Y) .
\end{aligned}
$$

The same holds if $\{X<-k\}=\{Y<-k\}$, because for all $\lambda \in[0,1]$ we have $\{X<-k\}=$ $\{\lambda X+(1-\lambda) Y\}=\{Y<-k\}$.

Proposition 4.7 (Translation by a constant). Suppose that $X \in\left[x^{\min }, x^{\max }\right]$ for $x^{\max }, x^{\min }$ constants and we take $m$ constant.

(1) For moderate values of $m$, namely: if $-v\left(k+x^{m i n}\right) \leq m<0$ or $0<m \leq v^{-1}(m)-$ $\left(k+x^{\max }\right)$, then:

$$
S R M(X+m) \geq S R M(X)-m
$$

15 
(2) For extreme values of $m$, namely: if $m<-v\left(k+x^{\max }\right)$ or $m>\max \left(v^{-1}(m)-(k+\right.$ $\left.\left.x^{\min }\right) ; 0\right)$, then:

$$
S R M(X+m) \leq S R M(X)-m
$$

Proof. Let us suppose that $m<0$.

If $k+X+m \geq 0$, then necessarily $k+X>0$ and $v(k+X)-v(k+X+m) \geq-m$ (because $v^{\prime}(x) \geq 1$ for $x \in \mathbb{R}_{+}$and we can use the mean value theorem). It follows that $S R M(X+m) \geq S R M(X)-m$.

If $k+X+m<0$, and $k+X \leq 0$ then $v(k+X)=v(k+X+m)=0$ and hence, $v(k+X)-v(k+X+m)=0<-m$. It follows in this case that $S R M(X+m)<S R M(X)-m$.

If $k+X+m<0$, and $k+X \in\left[0, v^{-1}(-m)\right]$ then $v(k+X)-v(k+X+m)=v(k+X)<-m$, therefore $\operatorname{SRM}(X+m)<S R M(X)-m$.

If $k+X+m<0$, and $k+X \in\left[v^{-1}(-m),-m\right]$ then $v(k+X)-v(k+X+m)=v(k+X)>$ $-m$, therefore $\operatorname{SRM}(X+m)>S R M(X)-m$.

Summarizing the above we get: If $m<0$ constant, then:

- If $X \geq v^{-1}(-m)-k$, then $\operatorname{SRM}(X+m) \geq S R M(X)-m$;

- If $X \leq v^{-1}(-m)-k$, then $\operatorname{SRM}(X+m) \leq S R M(X)-m$.

We now suppose that $m>0$.

If $v(k+X+m) \leq m$, then necessarily $k+X<0$ and $v(k+X)-v(k+X+m)=$ $-v(k+X+m) \geq-m$. It follows that $\operatorname{SRM}(X+m) \geq S R M(X)-m$.

If $v(k+X+m)>m$, and $k+X \leq 0$ then $v(k+X)-v(k+X+m)=-v(k+X+m)<-m$. It follows in this case that $\operatorname{SRM}(X+m)<S R M(X)-m$.

If $v(k+X+m)>m$, and $k+X>0$ then we also have $v(k+X)-v(k+X+m)<-m$ (since $v^{\prime}(x) \geq 1$ for $x \in \mathbb{R}_{+}$and we can use the mean value theorem) hence $\operatorname{SRM}(X+m)<$ $\operatorname{SRM}(X)-m$.

Summarizing for $m>0$, we get:

- If $X \geq v^{-1}(m)-m-k$, then $\operatorname{SRM}(X+m) \leq S R M(X)-m$;

- If $X \leq v^{-1}(m)-m-k$, then $\operatorname{SRM}(X+m) \geq S R M(X)-m$.

Combining the results for $m>0$ and $m<0$ we get the result stated in the proposition.

One can notice that the following result was proved inside the proof of Proposition 4.7 (where in a first step we were not using upper and lower bounds for $X$ ):

Corollary 4.8. Suppose that $X \in \mathcal{X}_{k}$ and $m$ constant such that $X+m \in \mathcal{X}_{k}$. Then, the following hold:

(1) If $m<0$ then $\operatorname{SRM}(X+m) \geq S R M(X)-m$;

(2) If $m>0$ then $\operatorname{SRM}(X+m) \leq S R M(X)-m$.

The corollary above emphasizes that SRM fails to be translation invariant even when restricted to the case of sustainable risks. Intuitively, cash has more value when kept inside the company (except in the region where dividend distributions occur, as characterized in Proposition 4.3 (iv)).

The set $\mathcal{X}_{k}$ of sustainable risks has the convenient property of being a convex set. Therefore, we can construct a convex risk measure for $\mathcal{X}_{k}$ as follows. Let us denote by

$$
\mathcal{S}_{k}:=\left\{X \in \mathcal{X}_{k} \mid \operatorname{SRM}(X) \geq 0\right\}
$$


i.e., the set of sustainable and acceptable risks. This being also a convex set, we further denote for all $x \in \mathcal{X}_{k}$ :

$$
\rho_{\mathcal{S}}(X)=\inf \left\{m \geq-k \mid X \in \mathcal{S}_{k}\right\} .
$$

$\rho_{\mathcal{S}}$ is a convex risk measure (this can be checked using the Theorem 4.7 in [11]). The following result shows that the coherent measure $\rho_{\mathcal{S}}$ is more conservative, in the sense that it underestimates the non acceptable risks as compared with SRM.

Corollary 4.9. Let $X \in \mathcal{X}_{k}$ and $\rho_{\mathcal{S}}$ be as in (4.1). Then:

(1) If $X \in \mathcal{S}_{k}$ then $S R M(X) \leq \rho_{\mathcal{S}}(X)$.

(2) If $X \notin \mathcal{S}_{k}$ then $S R M(X) \geq \rho_{\mathcal{S}}(X)$.

Proof. Notice first that $\rho_{\mathcal{S}}$ is the unique solution for:

$$
\mathbf{E}[v(k+X+m)]=v(k) .
$$

That $\rho_{\mathcal{S}}$ solves (4.2) follows by dominated convergence, since the function $v$ is continuous and the solution is unique since $v$ is strictly increasing (indeed, since $X \in \mathcal{X}_{k}$ we need to consider the properties of the function $v$ on $\mathbb{R}_{+}$only).

As a consequence:

$$
\operatorname{SRM}\left(X+\rho_{\mathcal{S}}(X)\right)=0 .
$$

Consider that $X \in \mathcal{S}_{k}$. Then $\rho_{\mathcal{S}}(X) \leq 0$. Therefore, from Corollary 4.8, we obtain that:

$$
0=S R M\left(X+\rho_{\mathcal{S}}(X)\right) \geq S R M(X)-\rho_{\mathcal{S}}(X) \text {. }
$$

On the other hand, if $X \in \mathcal{S}_{k}$ then $\rho_{\mathcal{S}}(X) \geq 0$. Therefore, again from Corollary 4.8, we obtain that:

$$
0=S R M\left(X+\rho_{\mathcal{S}}(X)\right) \leq S R M(X)-\rho_{\mathcal{S}}(X)
$$

\section{Risk MEASURES ASSOCIATED WITH CASH FLOWS ARRIVING AT A FUTURE DATE}

Let us now assume that the random shock $X$ will affect the cash flow process at a future date $T \geq 0$, the risk is hence characterized by the couple $(T, X)$. We assume that the shareholders can implement dividend strategies optimally, anticipating the random shock $(T, X)$. In this case, the value function will be different from the one characterized in Section 3 and the definition of $S R M$ should be extended to reflect the anticipation of shocks that arrive in the future.

For simplicity, we only consider here the case where the risk $(T, X)$ has the characteristics:

(1) $T$ is a stopping time in the filtration $\left(\mathcal{F}_{t}\right)$ generated by the cash process $\left(C_{t}\right)$.

(2) $X$ is a random variable independent from $\mathcal{F}_{\infty}$.

We now define the cash process in presence of the random shock $(T, X)$ and a dividend process $\left(\xi_{t}\right)$, as a stochastic process $\left(\tilde{C}_{t}\right)$ with dynamics:

$$
\begin{array}{ll}
d \tilde{C}_{t} & =d C_{t}+X d \mathbf{1}_{\{T \leq t\}} \quad \text { for } t \in[0, \infty) \\
\tilde{C}_{0^{-}} & =k
\end{array}
$$

The dependence in the chosen dividend strategy $\xi$ is implicit via the process $C$. 
We denote by $\left(\mathcal{G}_{t}\right)$ the natural augmented filtration of the process $\left(\tilde{C}_{t}\right)$. We have that $\mathcal{G}_{t}=\mathcal{F}_{t} \vee \sigma\left(X \mathbf{1}_{\{T \leq s\}}, s \leq t\right)=\mathcal{F}_{t} \vee \sigma\left(X \mathbf{1}_{\{T \leq t\}}\right)$ (the last equality holds because $T$ is considered a stopping time in the filtration $\left.\left(\mathcal{F}_{t}\right)\right)$. In particular $\mathcal{F}_{t}=\mathcal{G}_{t}$ for $t<T$. Let:

$$
\tilde{\tau}(t):=\inf \left\{s \geq t \mid \tilde{C}_{s}<0\right\}, \quad t \geq 0
$$

i.e., $\tilde{\tau}(t)$ is the first time after time $t$ when the cash process is negative, in presence of the random shock $(T, X)$. Similarily, we define for the process $\left(C_{t}\right)$, i.e., in absence of the random shock $(T, X)$ :

$$
\tau(t):=\inf \left\{s \geq t \mid C_{s}<0\right\}, \quad t \geq 0 .
$$

For simplicity, we write $\tilde{\tau}$ (respectively $\tau$ ) for $\tilde{\tau}(0)$ (respectively $\tau(0)$ ). The optimal dividend strategy after time $t$ is given as a solution of the problem:

$$
V_{t}:=\sup _{\xi \in \tilde{\mathcal{A}}_{t}} \mathbf{E}\left[\int_{[t, \tilde{\tau}(t)]} e^{-\rho(s-t)} d \xi_{s} \mid \mathcal{G}_{t}\right]
$$

with $\tilde{\mathcal{A}}_{t}=\tilde{\mathcal{A}}\left(t, \tilde{C}_{t}\right)$, where $\tilde{\mathcal{A}}(t, x)$ is the set of admissible controls after time $t$, that is, the set of $\left(\mathcal{G}_{t}\right)$-adapted, nondecreasing, càdlàg processes $\xi$ such that a unique strong solution $\left(\tilde{C}_{s}\right)_{s>t}$ exists conditionally on $\left\{\tilde{C}_{t^{-}}=x\right\}$, and such that for any stopping times $t \leq S \leq T$ we have: $\int_{S}^{T} \tilde{C}_{s} d \xi_{s} \geq 0$. This last condition ensures that no dividends are payed that would exceed the cash reserve available. We shall consider $\left(V_{t}\right)$ to be right-continuous.

For defining the dynamic version of the SRM, we need to write more precisely the value function before the random shock occurs. For this purpose, we introduce the two dimensional process $Z=\left(Z^{1}, Z^{2}\right), \mathbb{R}_{+} \times \mathbb{R}$-valued with components having the dynamics: $d Z_{s}^{1}=d s$, $d Z_{s}^{2}=d C_{Z_{0}^{1}+s}, s \geq 0$. We introduce a family of probability measures $\left(\mathbf{P}^{(t, x)}\right)_{\left(t \in \mathbb{R}_{+}, x \in \mathbb{R}\right)}$ on $\left(\mathbb{R}_{+} \times \mathbb{R} \times \Omega, \mathcal{B}\left(\mathbb{R}_{+} \times \mathbb{R}\right) \otimes \mathcal{F}\right)$ such that under $\mathbf{P}^{(t, x)}$, the process $Z$ starts at $(t, x)$ (i.e., $\left.Z_{0}^{1}=t, Z_{0}^{2}=x\right)$ ). We denote hereafter $\mathbf{E}^{(t, x)}$ the expectation under $\mathbf{P}^{(t, x)}$.

We also define:

$$
\tilde{v}(t, x):=\sup _{\xi \in \tilde{\mathcal{A}}(t, x)} \mathbf{E}^{(t, x)}\left[\int_{[0, \tilde{\tau}]} e^{-\rho s} d \xi_{s}\right] .
$$

By (slight) abuse of notation we have used above $\tilde{\tau}=\inf \left\{s \geq 0 \mid Z_{s}^{2}<0\right\}$.

We now give an expression of the process $V$ using the functions $v$ and $\tilde{v}$.

Proposition 5.1. The process $V$ is given by:

$$
V_{t}= \begin{cases}\tilde{v}\left(t, C_{t}\right) & \text { if } t<T \\ v\left(\tilde{C}_{t}\right) & \text { if } t \geq T\end{cases}
$$

Proof. Recall that for all $t<T$ we have that $\tilde{C}_{t}=C_{t}$ and therefore $\mathcal{F}_{t}=\mathcal{G}_{t}$ and $\tilde{\mathcal{A}}_{t}=$ $\tilde{\mathcal{A}}\left(t, C_{t}\right)$. Then:

$$
\tilde{v}\left(t, C_{t}\right)=\sup _{\xi \in \tilde{\mathcal{A}}\left(t, C_{t}\right)} \mathbf{E}\left[\int_{t^{-}}^{\tilde{\tau}(t)} e^{-\rho(s-t)} d \xi_{s} \mid \mathcal{G}_{t}\right]=V_{t}
$$

since it corresponds precisely to the problem in (5.3). 


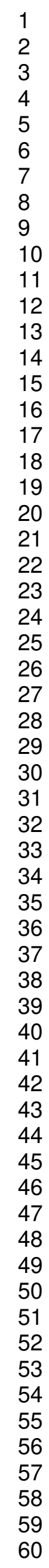

Mathematical Finance 


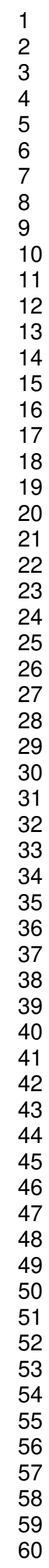




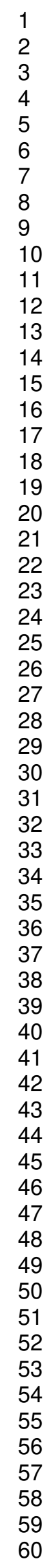




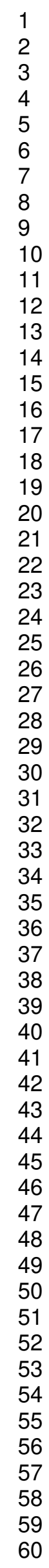




\title{
SHAREHOLDER RISK MEASURES
}

\author{
DELIA COCULESCU AND JEAN-CHARLES ROCHET
}

\begin{abstract}
The aim of this paper is to put forward a new family of risk measures that could guide investment decisions of private companies. But at the difference of the classical approach of Artzner, Delbaen, Eber and Heath (1999) and the subsequent extensions of this model, our risk measures are built to reflect the risk perception of shareholders rather than regulators. Instead of an axiomatic approach, we derive risk measures from the optimal policies of $\equiv$ eholder value maximizing company. We study these optimal policies and the related risk measures that we call shareholder risk measures. We emphasize the fact that due to the specific corporate environment, in particular the limited shareholders' liability and the possibility to pay out dividends from the cash reserves, these risk measures are not convex. Also, they depend on the specific economic situation of the firm, in particular its current cash level, and thus they are not translation invariant. This paper bridges the gap between two important branches of mathematical finance: risk measures and optimal dividends.
\end{abstract}

\section{INTRODUCTION}

The aim of this paper is to put forward a new family of risk measures that are associated with the optimal policies of a shareholder value maximizing company. We call them shareholder risk measures. Due to the specific corporate environment, in particular the limited shareholders' liability and the possibility to pay out dividends from the cash reserves, these risk measures are not convex. Also, they depend on the specific economic situation of the firm, in particular the current cash level, and thus they are not translation invariant. Our shareholder risk measures capture the privately optimal behavior of a company, from the point of view of its shareholders. By contrast, traditional risk measures, that were developed after the seminal work of Artzner and al. [3], adopt a normative approach, typically what a regulator would impose to the company (for example in the form of a capital requirement), with the aim of limiting the probability of failure.

In the work by Artzner and al. [3], a risk measure $\rho$ is defined using a set of axioms, which represent some properties that a risk measure should have in an abstract set-up: $\rho$ is a functional that assigns a real value to a random variable (the risk), it is positively homogeneous, subadditive, translation invariant (also called cash invariant in this context) and monotone.

Department of Banking and Finance, Universität Zürich, Plattenstrasse 32, Zürich 8092, SWITZERLAND.

E-mail addresses: delia.coculescu@bf.uzh.ch, jean-charles.rochet@bf.uzh.ch.

Date: 4 december 2014.

2000 Mathematics Subject Classification. 15A52.

Key words and phrases. Risk measures, shareholder's risk measure, cash reserves management, dividend optimization, non-convex risk measures.

The research leading to these results has received funding from the European Research Council under the European Community's sevenths Framework Programme (FP7/2007-2013) grant agreements and from NCCR Finrisk (project Banking and Regulation). 
Such risk measures are called coherent. An important extension of the concept of coherent risk measures is the notion of convex risk measures, developed simultaneously by Föllmer and Schied [14] and Fritelli and Rosazza-Gianin [16], who proposed to relax the properties of subadditivity and positive homogeneity of $\rho$ with the weaker property of convexity:

$$
\rho(\lambda X+(1-\lambda) Y) \leq \lambda \rho(X)+(1-\lambda) \rho(Y), \quad \forall \lambda \in[0,1]
$$

Our aim in this paper is to develop new risk measures, that share some similar properties with the convex risk measures: they create an ordering on random cash flows which is consistent with preferences of a class of agents (here the shareholders of a company) and are expressed in monetary units. But, at the difference of the axiomatic approach of Artzner and al. [3] and its subsequent extensions, our risk measures are derived from the dividend policy - cash optimization problem faced by a firm under uncertainty. We thus make a bridge been two different problems in corporate finance, namely the optimal dividend policy and the measurement of risks.

In the dividend policy literature, given the characteristics of the cash flow process of a firm, two fundamental questions are addressed: when should dividends be distributed, and how much of the available cash should be distributed. The answer to these questions is called a dividend strategy. The classical approach, initiated by de Finetti [11], is to maximize the expected present value of all (future) dividends that will be distributed until the company is ruined (we shall call this quantity the shareholder value). A rich literature has investigated this important topic (see a selection in Subsection 3.2 below).

Assuming that the objective of the firm is to maximize shareholder value examine in this paper the impact of a random shock $X$ on the firm's cash reserves on shareholder value and use it as a measure of the risk of the random shock $X$. The properties of the resulting risk measures are aftern $\overline{\bar{\nu}}$ s analyzed. This approach has the advantage that it displays some specific features of the risk measures, such as the non convexity or the absence of translation invariance. Even though model dependent, our approach helps to understand from where these atypical and perhaps at first sight non-intuitive features of our proposed risk measures come from: we show that the particular corporate environment, such as limited liability for shareholders and the possibility to pay dividends from the available cash, lead to such specific risk measures. Of course, in practice shareholder value depends on many other variables than cash reserves, such as assets profitability, growth opportunities, macroeconomic context. We abstract from these exogenous variables for the sake of clarity.

The remaining of the paper is organized as follows: Section 2 summarizes important concepts and definitions, Section 3 introduces the shareholder value maximization problem and characterizes the value function and the optimal dividend strategies. In Section 4 we establish the main properties of the SRM as opposed to the convex risk measures. Section 5 is an introduction to the dynamic SRM, i.e., when the random shock affects the cash reserves at some future date.

\footnotetext{
${ }^{1}$ This is indeed the mandate given to the manager by the owners of the firm. We leave aside agency conflicts, i.e., what happens when the manager pursues its own objectives at the expense of shareholders.
} 


\section{Some Useful Definitions}

The dividend policy - cash optimization problem which will be at the base of our discussion gives raise $\equiv$ a value function that is not concave everywhere. It is useful therefore to introduce from now the concept of shareholder value function as:

Definition 2.1. A shareholder value function is a real valued function $v$ that maps the level of the cash reserves of the firm to its shareholder value, it is increasing, nonnegative and satisfies $v(x) \geq x$.

The function $v$ expresses the preference for more rather than less (by the property of being increasing), the shareholder's limited liabilities (by the non negativity of the function). The last property is an expression of the fact that shareholders are owners and can decide to liquidate a company if the cash value is higher. We shall develop later more economic foundations for this shareholder value function.

Let $\mathcal{X}$ be a set of random variables on the probability space $(\Omega, \mathcal{F}, \mathbf{P})$. A risk measure is a mapping $\rho$ from a $\mathcal{X}$ to the real line. Let us now review two common ways of measuring a risk $X$ and then propose a third method which will be the one we shall study in this paper. In all cases, we use a shareholder value function $v$, and we suppose that an agent (the firm in our case) has already an available cash flow $k$. In order to emphasize the links with the utility theory of von Neumann and Morgenstern we introduce the function:

$$
u_{k}(x):=v(k+x)-v(k) .
$$

(1) Indifference price:

$$
\rho_{k}(X):=\inf \left\{m \mid \mathbf{E}\left[u_{k}(X+m)\right] \geq 0\right\}
$$

This is a generalization of the classical approach of shortfall risk measures seen as the distance to some convex acceptable set of random variables. Indeed, we can define the following acceptance set $\mathcal{A}_{k}:=\left\{x \in \mathcal{X} \mid \mathbf{E}\left(\ell_{k}(-X)\right] \leq 0\right\}$, with $\ell_{k}(x)=-u_{k}(-x)$. Then $\rho_{k}(X)=\inf \left\{m \mid X+m \in \mathcal{A}_{k}\right\}$ as in [3], but here $\mathcal{A}_{k}$ is not convex when $v$ is not concave.

Also, let us note that, on the level sets where $v$ is strictly increasing and concave, $\rho$ can be seen as the indifference price from the expected utility theory, since it is the unique solution $m$ of the equation: $\mathbf{E}\left[u_{k}(m+X)\right]=u_{k}(0)=0$. But $\rho$ can also be (and is commonly) interpreted as a capital requirement.

(2) Certainty equivalent:

$$
C E_{k}(X):=-\inf \left\{m \mid \mathbf{E}\left[u_{k}(X)\right] \leq u_{k}(m)\right\} .
$$

This measure corresponds to the concept of the certainty equivalent for $X$, i.e., the sure amount for which an agent remains indifferent to a lottery $X$. Indeed, if we suppose again that $v$ is concave so that $u_{k}(x)=v(k+x)-v(k)$ can be interpreted as a utility function in a classical sense, then $C E_{k}(x)=u_{k}^{-1} \mathbf{E}\left[u_{k}(X)\right]$, where $u_{k}^{-1}$ is the left-continuous inverse of $u_{k}$.

We put forward a new class of risk measures:

(3) Impact price, or shareholder risk measure:

$$
S R M_{k}(X):=v(k)-\mathbf{E}[v(k+X)]=-\mathbf{E}\left[u_{k}(X)\right]
$$


This is a measure of the loss in shareholder value that a random cash flow produces, supposing shareholder value function is $v$. We shall endogenize the function $v$ for the shareholder value maximization. Note that $S R M$ is conceptually different from the measures presented above in (1) and (2). It is related to the utility theory of von Neumann and Morgenstern with the following notable difference that the "utility" function we use:

(a) is not a concave function;

(b) depends on the current cash level in the firm. This means that as the cash level of the firm evolves, the "utility" function changes. Since the cash evolution is random, the utility function that will apply at a future date is a random variable;

(c) can be extended to a dynamic set-up.

(d) represents a price, i.e., can be expressed in monetary units;

We can already remark that the fact that the cash invariance property fails, is a consequence of the fact that $S R M$ is not defined as a distance to some acceptable set, as is the case of the coherent (and convex) risk measures, the financial interpretation being that cash has sometimes more value inside the company than in the shareholder's pocket (or sometimes the opposite). The nonconvexity of $S R M$ is linked to the nonconcavity of the function $v$. As the set of acceptable risks $\mathcal{A}_{k}:=\{X \mid S R M(X) \leq 0\}$ is not convex, a risk measure in the spirit of [3] is also non convex. At fixed level of the cash $k$ in the firm, we shall nevertheless identify some classes of risks for which $S R M$ is convex or translation invariant (within a given range of the cash being added/subtracted).

It can seem at a first sight surprisingeto have risk measures that may penalize diversification. Quasi-concavity of risk measures seems to be a very natural feature, and $S R M$ do not satisfy this property in general. Let us emphasize again that here we do not take the point of view of regulators, nor of a manager of some given portfolio in a company. From the shareholders' perspective, it is not always preferable to diversify risks involving both some positive payments and, as a downside, the default of the company. If we exclude these risks, we obtain what we shall call the class of sustainable risks, i.e., all random payments that do not produce the default of the company. $S R M$ is convex for the sustainable risks.

Finally, let us point out that it is of course possible to construct a robust version of the SRM by using a set of scenarios (i.e., probabilities measures) $\mathcal{Q}$ as:

$$
S R M_{k}(X):=\max _{\mathbf{Q} \in \mathcal{Q}} \mathbf{E}_{\mathbf{Q}}\left[-u_{k}(X)\right],
$$

this being perfectly compatible with the model we describe below (including in the dynamic version of SRM).

\section{Shareholder VAlue maximization}

This section presents the problem of optimal cash management of a firm optimizing the flow of dividends p value function. We suppose that in sence of dividend payments, the available cash reserve of a firm follows, as time goes on, a jump diffusion. At any time, as long as the cash reserve remains positive, the firm can decide to pay a part of the available cash as dividends. The objective is to determine the dividend scheme that maximizes the expected discounted flow of dividends until the bankruptcy time (which is the first time when the cash process is less than zero). 
The mathematical formulation is as follows. We suppose that $\left(\Omega, \mathcal{F},\left(\mathcal{F}_{t}\right)_{t \geq 0}, \mathbf{P}\right)$ is a filtered probability space and $\left(W_{t}\right)$ a standard Brownian motion. Let $\tilde{N}$ be an $\left(\mathcal{F}_{t}\right)$ compensated Poisson random measure,

$$
\tilde{N}(t, A)=N(t, A)-\mathbf{E}[N(t, A)]=N(t, A)-\nu(A) t
$$

where for every Borel set $A, N(t, A)$ is a Poisson random measure measuring the number of jumps with amplitude in $A$, up to and including time $t$ and $\nu$ is the Lévy measure associated with $N$. Here, $\nu$ is assumed finite and admiting a monotone density on $\mathbb{R}_{-}$.

Starting from an initial state $k \in \mathbb{R}$ for the cash level and given a dividend policy $\left(\xi_{t}\right)$ (i.e., our control process), the dynamics of the cash reserve process of the firm is governed by:

$$
\begin{aligned}
& d C_{t}=b\left(C_{t}\right) d t+\sigma d W_{t}+\int_{\mathbb{R}} z \tilde{N}(d t, d z)-d \xi_{t}, \\
& C_{0^{-}}=k
\end{aligned}
$$

with $b(x)=\mu+r x$. The function $b$ corresponds to the sum of expected earnings and interests paid on the cash reserves $\left(C_{t}\right)$. The process $\left(\xi_{t}\right)$ is an adapted increasing process, right-continuous, with $\xi_{0^{-}}=0$, representing the cumulated dividend payments until time $t$ (our control process). Hence, the dynamics of the process in (3.1) represents the economically meaningful evolution of cash reserves.

As it is common in the theory of Markov processes, we introduce a family of probability measures $\left(\mathbf{P}^{x}\right)_{x \in \mathbb{R}}$, corresponding to a translation of $\left(C_{t}\right)$ such that $C_{0-}=x$ under $\mathbf{P}^{x}$ and thus allow for having arbitrary initial conditions. We write $\mathbf{P}^{k}=\mathbf{P}$. We denote $\mathbf{E}^{x}$ for the expectation operator under the measure $\mathbf{P}^{x}$.

Let:

$$
\tau=\inf \left\{t \geq 0 \mid C_{t}<0\right\}
$$

be the time of bankruptcy of the firm. Obviously $\left(C_{t}\right)$ and $\tau$ depend on the chosen dividend policy $\left(\xi_{t}\right)$. We use the simple notations that do not make explicit this dependence and believe that this does not create any confusions. Let

$$
J^{\xi}(x)=\mathbf{E}^{x}\left[\int_{0^{-}}^{\tau} e^{-\rho t} d \xi_{t}\right], \quad \rho>0 \text { constant. }
$$

be the expected total discounted amount of dividends phareholders up to the bankruptcy time. The notation $\int_{0^{-}}^{\tau}$ stands for $\int_{[0, \tau]}$. The discount rate $\rho$ is constant.

We assume that the probability measure $\mathbf{P}$ is the risk-adjusted probability measure. Thus, shareholders are supposed to be able to diversify their portfolios. Our model corresponds to a large company with diffuse ownership. It does not apply to a small company that would be owned and managed by the same agent. In that case, the risk aversion of this owner-manager would appear in the maximization problem. For such an analysis, look for example at Bank an Riedel [7].

We define the set of admissible controls $\mathcal{A}$ as the set of adapted, nondecreasing, càdlàg processes $\xi$ such that a unique strong solution $\left(C_{t}\right)$ of (3.1) exists, and such that for any stopping times $S \leq T$ we have: $\int_{S}^{T} C_{s} d \xi_{s} \geq 0$. This last condition ensures that no dividends are payed that would exceed the cash reserve available. 
We assume that the objective of the firm is to implement such a dividend policy $\xi^{*}$ that maximizes the function $J^{\xi}$, that is:

$$
v(x):=\sup _{\xi \in \mathcal{A}} J^{\xi}(x)=J^{\xi^{*}}(x) .
$$

In the remaining of this section we aim to emphasize some properties of the function $v$ which are useful later on, in particular the fact that it is not a concave function on $\mathbb{R}$ but it is concave on $\mathbb{R}_{+}$.

Let us introduce the characteristic operator $\mathbb{L}_{C}$ defined on a function $F: \mathbb{R} \rightarrow \mathbb{R}$ as follows:

$$
\mathbb{L}_{C} F(x)=\lim _{U \downarrow x} \frac{\mathbf{E}^{x} F\left(C_{T_{U}}\right)-F(x)}{\mathbf{E}^{x} T_{U}}
$$

where the limit is taken over a family of open sets $U$ shrinking down to $x$ in $\mathbb{R}$ and $T_{U}=$ $\inf \left\{t \geq 0 \mid C_{t} \in U^{c}\right\}$. From the general theory of Markov processes we know that the characteristic operator $\mathbb{L}_{C}$ that ${ }_{\overline{2}}$ sponds the jump diffusion appearing in (3.1) when no dividends are pa $\equiv$ (that is $\xi \equiv 0$ ) and applied to a $C^{2}(\mathbb{R})$ function $\phi$ takes the following integro-differential form:

$$
\mathbb{L}_{C} \phi(x)=b(x) \frac{\partial \phi}{\partial x}(x)+\frac{1}{2} \sigma^{2} \frac{\partial^{2} \phi}{\partial x^{2}}(x)+\int_{\mathbb{R}}\left(\phi(x+z)-\phi(x)-\frac{\partial \phi}{\partial x}(x) z\right) \nu(d z)
$$

Also, it will be useful to recall the fellowing definition for the local time appearing in the $\times$ solution of the Skorohod problem for jump diffusions:

Definition 3.1. Let $O \subset \mathbb{R}$ be an open set. The Skorohod problem for the reflected jump diffusion $C$ into $\bar{O}$ is to find a pair $\left(C_{t}, \xi_{t}\right)$ of càdlàg adapted processes such that the following eonditions are satisfied:

$$
\begin{aligned}
& d C_{t}=b\left(C_{t}\right) d t+\sigma d W_{t}+\int_{\mathbb{R}} z \tilde{N}(d t, d z)-d \xi_{t}, \\
& C_{t} \in \bar{O}, \forall t \geq 0, \\
& \left(\xi_{t}\right) \text { has finite variation and } d \xi_{t}=0 \text { if } C_{t} \in O .
\end{aligned}
$$

The process $\xi$ is called the local time at the boundary $\partial O$ of the reflected process $C$.

3.1. A characterization of the optimal dividend strategy. The theorem below states some properties of the value function when it is sufficiently smooth $\$$ and of the corresponding optimal dividend strategy. The following conditions are assumed to hold:

(A1) $\mu>\int_{\mathbb{R}} z \nu(d z)$.

(A2) $\rho>r$.

(A3) $\sigma \neq 0$.

The case $\sigma=0$ is treated in Albrecher and Thonhauser [2]. Conditions (A1)-(A3) all play an important role in establishing the next result; additionally, the first two conditions can be interpreted as some profitability conditions for the firm. 
Theorem 3.2. Suppose that there exists a function $\phi \in C^{2}((0, \infty)) \cap C(\mathbb{R})$ such that $\phi$ solves the following variational inequations:

$$
\begin{aligned}
\sup \left\{\mathbb{L}_{C} \phi(x)-\rho \phi(x), 1-\frac{\partial \phi}{\partial x}(x)\right\} & =0, \quad \forall x \in(0, \infty) \\
\phi(x)=0, & \forall x \leq 0,
\end{aligned}
$$

with the set $\left\{x \in(0, \infty) \mid \mathbb{L}_{C} \phi(x)-\rho \phi(x)<0\right\}$ being non empty.

Then:

(i) There exists a constant $x^{*} \geq 0$ such that:

$$
O:=\left\{x \in(0, \infty) \mid \mathbb{L}_{C} \phi(x)-\rho \phi(x)=0 \text { and } \frac{\partial \phi}{\partial x}(x)>1\right\}=\left(0, x^{*}\right)
$$

(the non intervention set).

(ii) $\phi$ is concave on the interval $[0, \infty)$.

(iii)

$$
v(x)=\phi(x)
$$

(iv) The optimal control $\xi^{*}$ is the local time at $x^{*}$ of the jump diffusion $C$ reflected back into $\left(0, x^{*}\right]$.

Proof. In this proof, we se shall always use the notation $\phi^{\prime}(0)$ for the right-hand derivative at zero.

Let us introduce the following set:

$$
D:=\left\{x \in(0, \infty) \mid \frac{\partial \phi}{\partial x}(x)=1 \text { and } \mathbb{L}_{C} \phi(x)-\rho \phi(x) \leq 0\right\}
$$

$O$ and $D$ are disjoint sets. Moreover, by definition of $\phi$ we have $O \cup D=(0, \infty)$. $O$ is open and the interior of $D$ is not empty (since the set $\left\{x \in(0, \infty) \mid \mathbb{L}_{C} \phi(x)-\rho \phi(x)<0\right\}$ is supposed non empty).

Let us prove (i). If $\mu=b(0) \leq 0$ then $\phi(x)=x \vee 0$ is a solution of (3.7), (3.8) and $D=(0, \infty)$, hence in this case (i) and (ii) hold.

If $\mu>0$, one can check that $0 \notin \bar{D}$. We remark that whenever $x$ belongs to the interior of $D$ then $[x, \infty) \subseteq D$. Indeed, in $D, \mathbb{L}_{C} \phi(x)-\rho \phi(x)=b(x)-\rho(x+K):=h(x)$ with $K$ some constant such that $h(x) \leq 0$. The function $h$ is strictly decreasing since $b^{\prime}(x)<\rho$ for every $x$, hence the statement. In order to show that $D=\left[x^{*}, \infty\right)$, for some $x^{*}>0$, it remains to show that $D$ does not have isolated points, other configurations for $D$ being already excluded by the above remark. For this, it is sufficient to show that $\phi^{\prime}$ is monotonic, $D$ being precisely the set where $\phi^{\prime}$ attains its minimum. Below we show that $\phi$ is concave, hence $\phi^{\prime}$ monotonic.

We now prove (ii), that is, $\phi$ is concave. We treat the case $\mu>0$ (we have shown that when $\mu \leq 0$ then $\phi$ is linear, hence concave).

In $O, \phi$ satisfies:

$$
\frac{1}{2} \sigma^{2} \frac{\partial^{2} \phi}{\partial x^{2}}(x)=\rho \phi(x)-b(x) \frac{\partial \phi}{\partial x}(x)-\int_{\mathbb{R}}\left(\phi(x+z)-\phi(x)-\frac{\partial \phi}{\partial x}(x) z\right) \nu(d z):=g(x) .
$$

The function $g$ satisfies $g(0)<0$ (due to our assumption $(\mathrm{A} 1)$ ), hence $\phi$ 元ncave in $O$ near 0 . In other words, $\phi^{\prime}$ satisfies $\phi^{\prime}(0)>\phi^{\prime}\left(x^{*}\right)=1$ and is $\phi^{\prime}$ is decreasing near 0 . 
Suppose that $\phi^{\prime}$ is not monotonic. Then there must be at least a local minimum of $\phi^{\prime}$ inside $\left(0, x^{*}\right)$, at a point say $x_{m}$ and a local maximum at a point say $x^{m}$ with $0<x_{m}<x^{m}<x^{*}$. Without loss of generality, we assume that $x_{m}$ is the first local minimum of $\phi^{\prime}$, i.e., $\phi^{\prime}$ is decreasing on the interval $\left(0, x_{m}\right]$. Furthermore, and also without loss of generality, we suppose $\phi^{\prime}\left(x^{m}\right)$ is the maximum value of $\phi^{\prime}$ that also satisfies $\phi^{\prime \prime}=0$ (i.e., $\phi^{\prime}\left(x^{m}\right)$ is a global maximum for $\phi^{\prime}$ inside the interval $\left.\left(x_{m}, x^{*}\right)\right)$. Our aim in what follows is to show $\phi^{\prime}$ cannot have a local maximum at $x^{m}$, which is a necessary and sufficient condition for proving the monotonicity of $\phi^{\prime}$ hence the concavity of $\phi$.

The sign of $\phi^{\prime \prime}$ is given by the sign of the function $g$ in (3.9), since by the assumption (A3), $\sigma \neq 0$. Hence, we need to have:

$$
g^{\prime}\left(x^{m}\right)<0
$$

and:

$$
g^{\prime}(x)>0 \text { for } x \in\left\{x_{m}, x^{*}\right\} .
$$

Using (A2) and the properties: $\phi^{\prime \prime}\left(x_{m}\right)=\phi^{\prime \prime}\left(x^{m}\right)=\phi^{\prime \prime}\left(x^{*}\right)=0$ and $\phi(0)=0$, we get:

$$
g^{\prime}(x)=(\rho-r) \phi^{\prime}(x)+\int\left(\phi^{\prime}(x)-\phi^{\prime}(x+z)\right) \nu(d z) \text { for } x \in\left\{x_{m}, x^{m}, x^{*}\right\} .
$$

Since $\rho>r$, we see that the maximum of $\phi^{\prime}$ needs to be attained at the boundary, i.e., at $x=0$ and $x^{m}$ can only be a local maximum of $\phi^{\prime}$ (otherwise (3.10) cannot be fulfilled).

The required inequalities (3.10) and (3.11) have in fact implications on the measure $\nu$. For instance, (3.10) cannot hold if the support of $\nu$ is contained in $\mathbb{R}_{+}$. Let us investigate these inequalities in more detail. Suppose that $\nu$ admits a nondecreasing density on $\mathbb{R}_{-}$. Substracting (3.11) from (3.10) with $x=x_{m}$, we obtain:

$$
\begin{aligned}
(\rho-r)\left(\phi^{\prime}\left(x^{m}\right)-\right. & \left.\phi^{\prime}\left(x_{m}\right)\right)<\int\left(\phi^{\prime}\left(x^{m}+z\right)-\phi^{\prime}\left(x^{m}\right)\right)-\left(\phi^{\prime}\left(x_{m}+z\right)-\phi^{\prime}\left(x_{m}\right)\right) \nu(d z) \\
& <\int_{(-\infty, 0]}\left(\phi^{\prime}\left(x^{m}+z\right)-\phi^{\prime}\left(x^{m}\right)\right)-\left(\phi^{\prime}\left(x_{m}+z\right)-\phi^{\prime}\left(x_{m}\right)\right) \nu(d z)<0 .
\end{aligned}
$$

But this is a contradiction, since obviously we must have $(\rho-r)\left(\phi^{\prime}\left(x^{m}\right)-\phi^{\prime}\left(x_{m}\right)\right)>0$ (both $\rho>r$ and $\phi^{\prime}\left(x^{m}\right)>\phi^{\prime}\left(x_{m}\right)$ must hold). If $\nu$ admits ath nonincreasing density on $\mathbb{R}_{-}$ we substract again (3.11) from (3.10) but this time with $x^{*}$ instead of $x_{m}$ and we obtain identical inequalities as above and $\bar{\equiv}$ e contradiction.

This proves that a local maximum for $\phi^{\prime}$ inside $\left(0, x^{*}\right)$ cannot exist, i.e., $\phi^{\prime \prime}$ cannot switch the sign from being positive to being negative in $\left(0, x^{*}\right)$. Hence, $\phi^{\prime}(x)$ is monotonically decreasing on $\left[0, x^{*}\right)$, i.e., $\phi$ is concave on $\left[0, x^{*}\right)$.

(ii) is a consequence of the fact that $\phi$ is $C^{2}$ concave on $\left(0, x^{*}\right)$ and linear on $\left(x^{*}, \infty\right)$.

The proof of (iii) is classical. Suppose that $x>0$. Choose any dividend policy $\xi \in \mathcal{A}$ and $t$ in the stochastic interval $[0, \tau]$. By Itô's formula for the semimartingale $C$ (recall that $\phi$ is assumed to be $\left.C^{2}(0, \infty)\right)$ we have:

$$
\begin{aligned}
e^{-\rho t} \phi\left(C_{t}\right)= & \phi(x)+\int_{0}^{t} e^{-\rho s}\left(\mathbb{L}_{C} \phi\left(C_{s}\right)-\rho \phi\left(C_{s}\right)\right) d s+\int_{0}^{t} e^{-\rho s} \sigma \frac{\partial \phi}{\partial x}\left(C_{s}\right) d W_{s} \\
& -\int_{0}^{t} e^{-\rho s} \frac{\partial \phi}{\partial x}\left(C_{s-}\right) d \xi_{t}^{c}+\sum_{0<t_{n}<t} e^{-\rho t_{n}} \Delta_{\xi} \phi\left(C_{t_{n}}\right)
\end{aligned}
$$


where $\left(t_{n}\right)$ are the jumping times of $\xi$ and $\left.\Delta_{\xi} \phi\left(C_{t_{n}}\right)=\phi\left(C_{t_{n}}\right)-\phi\left(C_{t_{n}}+\Delta \xi_{t_{n}}\right)\right)$ is the variation of $\phi$ due to the jump $\Delta \xi_{t_{n}}=\xi_{t_{n}}-\xi_{t_{n}-}$. Since $\phi$ is increasing and $\xi$ increasing, all $\Delta_{\xi} \phi\left(C_{t_{n}}\right) \leq 0$.

Therefore, using (3.7):

$$
0 \leq e^{-\rho t} \phi\left(C_{t}\right) \leq \phi(x)+\int_{0}^{t} e^{-\rho s} \sigma \frac{\partial \phi}{\partial x}\left(C_{s}\right) d W_{s}
$$

The process $m_{t}:=\int_{0}^{t} e^{-\rho s} \sigma \frac{\partial \phi}{\partial x}\left(C_{s}\right) d W_{s}, t \geq 0$ is a local martingale. We take a localizing sequence $\left(T_{a}\right)_{a \geq 1}$ of this local martingale. We then define the increasing sequence of stopping times $\tau_{a}:=T_{a} \wedge \tau, a \geq 1$. Since the sequence $\left(m_{\tau_{a}}\right)$ is uniformly integrable, it follows from the above inequalities that also the sequence $\left(e^{-\rho \tau_{a}} \phi\left(C_{\tau_{a}}\right)\right)$ is uniformly integrable.

Taking expected value in (3.12) with $t=\tau_{a}$, we obtain:

$$
\begin{aligned}
\mathbf{E}^{x}\left[e^{-\rho \tau_{a}} \phi\left(C_{\tau_{a}}\right)\right]= & \phi(x)+\mathbf{E}^{x}\left[\int_{0}^{\tau_{a}} e^{-\rho t}\left(\mathbb{L}_{C} \phi\left(C_{t}\right)-\rho \phi\left(C_{t}\right)\right) d t-\int_{0}^{\tau_{a}} e^{-\rho t} \frac{\partial \phi}{\partial x}\left(C_{t-}\right) d \xi_{t}^{c}\right. \\
& \left.+\sum_{0<t_{n}<\tau_{a}} e^{-\rho t_{n}} \Delta_{\xi} \phi\left(C_{t_{n}}\right)\right] .
\end{aligned}
$$

From the inequations (3.7) we have that $\mathbb{L}_{C} \phi(x)-\rho \phi(x) \leq 0$. It follows that:

$$
\phi(x) \geq \mathbf{E}^{x}\left[e^{-\rho \tau_{a}} \phi\left(C_{\tau_{a}}\right)\right]+\mathbf{E}^{x}\left[\int_{0}^{\tau_{a}} e^{-\rho t} \frac{\partial \phi}{\partial x}\left(C_{t-}\right) d \xi_{t}^{c}-\sum_{0<t_{n}<\tau_{a}} e^{-\rho t_{n}} \Delta_{\xi} \phi\left(C_{t_{n}}\right)\right] .
$$

By the mean value theorem we have

$$
\Delta_{\xi} \phi\left(C_{t_{n}}\right)=-\frac{\partial \phi}{\partial x}\left(x_{n}\right) \Delta \xi_{t_{n}}
$$

for some point $x_{n}$ on the straight line between $C_{t_{n}}-\Delta \xi_{t_{n}}$ and $C_{t_{n}}$. Using the inequality $\frac{\partial \phi}{\partial x} \geq 1$, we obtain:

$$
\phi(x) \geq \mathbf{E}^{x}\left[e^{-\rho \tau_{a}} \phi\left(C_{\tau_{a}}\right)\right]+\mathbf{E}^{x}\left[\int_{0}^{\tau_{a}} e^{-\rho t} d \xi_{t}\right] .
$$

Letting $a \rightarrow \infty$, since $\left(\phi\left(C_{\tau_{a}}\right)\right)$ is uniformly integrable, $\lim _{a \rightarrow \infty} \tau_{a}=\tau$ and $\phi\left(C_{\tau}\right)=0$ a.s., we obtain that:

$$
\phi(x) \geq J^{\xi}(x)
$$

As this holds for any admissible control $\xi$, and since for $x \leq 0$ we have by definition $\phi(x)=$ $v(x)=0$, we conclude that:

$$
\phi(x) \geq v(x), \quad \forall x \in \mathbb{R} .
$$

In particular, since it is admissible to pay immediately time 0 dividends in amount $C_{0}$, $v(x) \geq x$ and hence we must also have:

$$
\phi(x) \geq x
$$

Now suppose that $\xi_{t}=L_{t}^{y}$, the local time at $y>0$ of the process $C$ reflected back into $(0, y]$, for some $y \geq 0$. Note that $L^{y} \in \mathcal{A}$. Indeed, $L^{y}$ is adapted, increasing, and càdlàg. Moreover, a unique strong solution of (3.1) when $\xi_{t}=L_{t}^{y}$ exists (see [10]).

Our aim is to show that there exists $x^{*} \geq 0$ such that the function $\phi(x):=\mathbf{E}^{x}\left[\int_{0}^{\tau} e^{-\rho t} d L_{t}^{x^{*}}\right]$ solves the problem (3.7)-(3.8) for every $x \in \mathbb{R}$. Combined with the inequality (3.13) this will prove that $L^{x^{*}}$ is the optimal strategy and $\phi=v$. 
For the moment take any $y>0$ and denote $g^{y}(x):=\mathbf{E}^{x}\left[\int_{0}^{\tau} e^{-\rho t} d L_{t}^{y}\right]$. Given $x \in(0, y)$, choose $\epsilon$ such that $(x-\epsilon, x+\epsilon) \in(0, y)$ and denote $T_{\epsilon}:=\inf \left\{t \geq 0 \mid C_{t} \in(x-\epsilon, x+\epsilon)^{c}\right\}$. By the strong Markov property we have:

$$
\mathbf{E}^{x} g^{y}\left(C_{T_{\epsilon}}\right)=\mathbf{E}^{x} \mathbf{E}^{C_{T_{\epsilon}}} \int_{0}^{\tau} e^{-\rho t} d L_{t}^{y}=\mathbf{E}^{x} e^{\rho T_{\epsilon}} \int_{T_{\epsilon}}^{\tau} e^{-\rho t} d L_{t}^{y}=\mathbf{E}^{x} e^{\rho T_{\epsilon}} \int_{0}^{\tau} e^{-\rho t} d L_{t}^{y}
$$

We have used the fact that $T_{\epsilon} \leq \tau$ a.s. and $\int_{0}^{T_{\epsilon}} e^{-\rho t} d L_{t}^{y}=0$. Hence, we see that:

$$
\begin{aligned}
\mathbb{L}_{C} g^{y}(x) & =\lim _{\epsilon \downarrow 0} \frac{\mathbf{E}^{x} g^{y}\left(C_{T_{\epsilon}}\right)-g^{y}(x)}{\mathbf{E}^{x} T_{\epsilon}}=\lim _{\epsilon \downarrow 0} \frac{\mathbf{E}^{x}\left(e^{\rho T_{\epsilon}}-1\right) \int_{0}^{\tau} e^{-\rho t} d L_{t}^{y}}{\mathbf{E}^{x} T_{\epsilon}} \\
& =\lim _{\epsilon \downarrow 0} \frac{\mathbf{E}^{x}\left(e^{\rho T_{\epsilon}}-1\right) \mathbf{E}^{x}\left(\int_{0}^{\tau} e^{-\rho t} d L_{t}^{y}\right)}{\mathbf{E}^{x} T_{\epsilon}}=\rho g^{y}(x) .
\end{aligned}
$$

This shows that $\mathbb{L}_{C} g^{y}(x)-\rho g^{y}(x)=0$ for $x \in(0, y)$. Also, by definition of $L^{y}$ we have that $g^{y}(y+c)=g^{y}(y)+c, \forall c>0$. Therefore, for any $x \in(y, \infty) g^{y}(x)$ is linear (hence $\left.C^{2}\right)$ and it can be checked that both $\frac{\partial g^{y}}{\partial x}(x)=1$ and $\mathbb{L}_{C} g^{y}(x)-\rho g^{y}(x)<0$ hold.

Let us notice that $g^{y}(x)=0$ for $x \leq 0$, that is equation (3.8) is verified by $g^{y}$.

In order to prove points (iii) and (iv) of the theorem it is now sufficient to show that $\exists x^{*}$ in $[0, \infty)$ such that $g^{x^{*}}$ is $C^{2}((0, \infty))$. But this fact is ensured by the fact that a $C^{2}$ solution to (3.7) is assumed to exist plus the previous remark that the set $O$ must be of the form $\left(0, x^{*}\right)$.

The next Corollary emphasizes the fact that the function $v$ is a shareholder value function (see Definition 2.1) and therefore the corresponding risk measures will not be convex. As we have shown, $v$ is concave on $(0, \infty)$, the concavity breaks down on $[-\infty, \infty]$ as a consequence of the existence of the limited liability for shareholders.

Corollary 3.3. Under the assumptions of Theorem 3.2, the value function $v(x)$ is a shareholder value function. It is constant on $\mathbb{R}_{-}$and concave on $\mathbb{R}_{+}$.

3.2. Some bibliographical comments and particular examples. There exists an extensive literature on barrier strategies for dividends, i.e., dividend payouts that are the local time of the cash process at an upper level. Let us mention that the book by Schmidli [27], treats among other topics the problem of dividend distributions in insurance companies and the reader can find an overview of the topic.

These models were initially developed by actuaries, where the cash process was considered to be the surplus of an insurance company. Dividends distributions of barrier type have been shown to be optimal for some types of cash processes. The first model of this type was elaborated by de Finetti [11]; he showed that if the risk/surplus process evolves as a random walk with step sizes \pm 1 , then an optimal way of paying out dividends is according to a barrier strategy. In the case of continuous-time models, the problem of finding the optimal dividend strategy has been studied extensively in the Cramér-Lundberg setting (Gerber [17]; Azcue and Muler [6]) and in the diffusion setting (Jeanblanc and Shiryaev [19]; Asmussen and Taksar [4]; Gerber and Shiu [18]). It was recently generalized to the spectrally negative Lévy model by Avram et al. [5], Kyprianou and Palmowski [20] and Loeffen [23], where the surplus is a general Lévy process with only negative jumps. 
In the Cramér-Lundberg setting, the cash process is described by a Lévy process without the Brownian part (this would correspond to our model with $r=\sigma=0$ ). Our condition (A3) was important in our proof of the Theorem 3.2 and it $m$ that our model does not fit the Cramér-Lundberg setting. Notice that Gerber [17] has shown that not only barrier but also band strategies can be optimal in the Cramér-Lundberg setting. A band strategy is such that the cash process is reflected at an upper barrier and absorbed to zero by paying a dividend once it crosses a lower barrier. This strategy can be optimal for some spectrally negative Lévy cash process (see also Azcue and Muler [6]) and for some models falling in the class studied in Abrecher and Thonhauser [2] (here again not containing a Brownian part).

We should emphasize that in the case of band strategies, the value function $v(x)$ is non concave even on $\mathbb{R}_{+}$, and also non smooth (in the sense that the first derivative is discontinuous). Concrete examples can be found for instance in Azcue and Muler [6] and Abrecher and Thonhauser [2]. In this case the value function remains however a shareholder value function (in the sense of our Definition 2.1) and a supremum of concave functions.

In the case of our model, the brownian component mathe cash entirely as a dividend triggers the default of the firm. Hence, we have the boundary condition $v(0)=0$. This property excludes to band strategies, as optimal strategies, even in the situations where the value function would not be smooth (i.e., $\left.C^{2}(0, \infty)\right)$.

Below we give some examples of cash processes and the associated value functions.

3.2.1. Brownian Motion. This model is presented in Jeanblanc-Picqué and Shiryaev [19] and Shreve, Lehoczky and Gaver [28]. Suppose $b(x) \equiv \mu>0$ and $N(t, A) \equiv 0$ for any Borel set $A$. In this case we obtain:

$$
v(x):=\frac{e^{r_{1} x}-e^{r_{2} x}}{r_{1} e^{r_{1} x^{*}}-r_{2} e^{r_{2} x^{*}}} \quad 0 \leq x \leq x^{*}
$$

where $x^{*}=\frac{1}{r_{1}-r_{2}} \log \frac{r_{2}^{2}}{r_{1}^{2}}$ and $r_{1}>0>r_{2}$ are the roots of the equation: $\sigma^{2} r^{2}+2 \mu r-2 \rho=0$. $v(x)=v\left(x^{*}\right)+x-x^{*}$ for $x>x^{*}$.

3.2.2. Linear drift. Suppose that $b(x)=\mu+r x$ and $N(t, A) \equiv 0$ for any Borel set $A$.

We obtain that $v$ is solution of:

$$
\begin{aligned}
\frac{\sigma^{2}}{2} v^{\prime \prime}(x)+(\mu+r x) v^{\prime}(x)-\rho v(x) & =0 \\
v(0) & =0 \\
v^{\prime}\left(x^{*}\right) & =1
\end{aligned}
$$

A change of variables: $f(y):=v(x) \exp \left(\frac{\sigma^{2}}{2}\left(r \frac{x^{2}}{2}+\mu x\right)\right)$, with $y=\sigma^{2}(\mu+r x)^{2} / 4 r$, leads to differential equation:

$$
y f^{\prime \prime}+\left(\frac{1}{2}-y\right) f^{\prime}-\lambda f=0
$$

with $\lambda:=\left(r-\rho \sigma^{2} / 2\right) / 2 r$.

We denote by $F(a, b, x)$ the standard confluent hypergeometric function (or Kummer's function) which arises as a solution of confluent hypergeometric differential equation $x y^{\prime \prime}+$ $(b-x) y^{\prime}-a y=0$. The solution of the equation (3.17) is:

$$
f(y)=C_{1} f_{1}(y)+C_{2} f_{2}(y)
$$




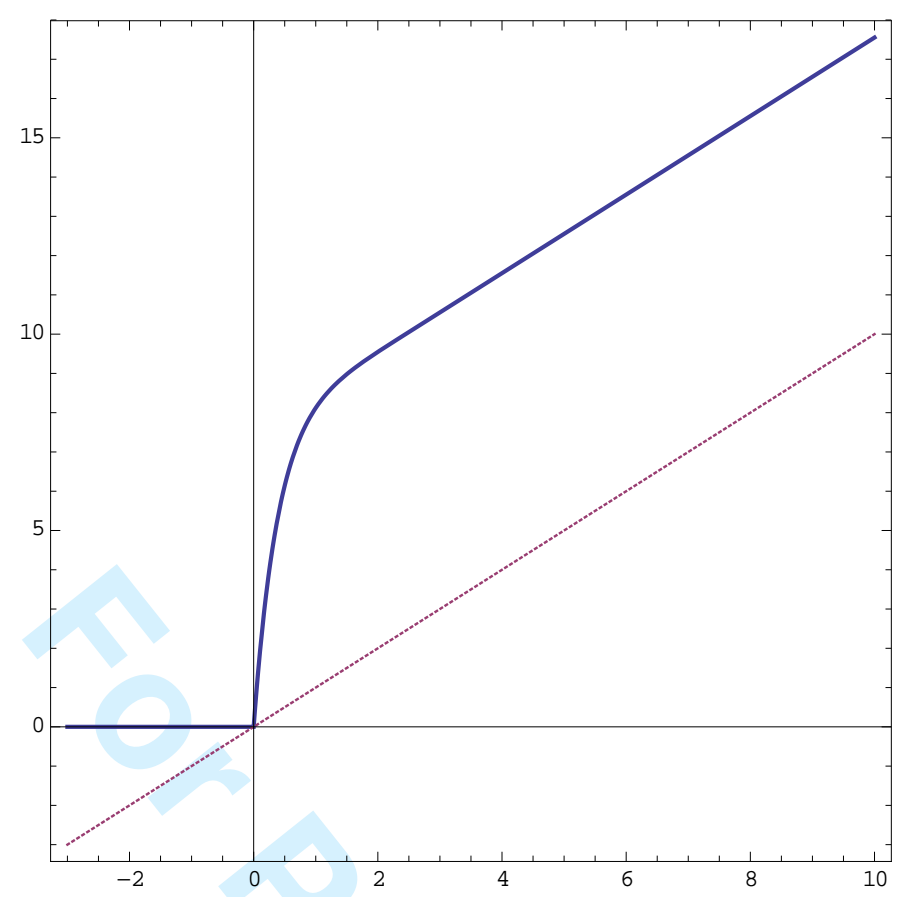

Figure 1. Value function $v(x)$ when the cash process $X$ is a Brownian Motion.

where $C_{1}$ et $C_{2}$ are constants and $f_{1}$ and $f_{2}$ are two independent solutions:

$$
\begin{aligned}
& f_{1}(y)=F(\lambda, 1 / 2, y) \\
& f_{2}(y)=y^{1 / 2} F(\lambda+1 / 2,3 / 2, y) .
\end{aligned}
$$

We obtain:

$$
v(x)=e^{\frac{\sigma^{2}}{2}\left(r \frac{x^{2}}{2}+\mu x\right)}\left(C_{1} F\left(\lambda, \frac{1}{2}, \frac{\sigma^{2}(\mu+r x)^{2}}{4 r}\right)+C_{2} \frac{\sigma(\mu+r x)}{2 \sqrt{r}} F\left(\lambda+\frac{1}{2}, \frac{3}{2}, \frac{\sigma^{2}(\mu+r x)^{2}}{4 r}\right)\right)
$$

(with the constants $C_{1}$ and $C_{2}$ fully determined by the boundary conditions).

3.2.3. Negative, exponentially distributed jumps. The case with $r=0, \nu(d z)=\mathbf{1}_{z<0} \lambda \delta e^{-\delta z} d z$ is studied in Belhaj [8] (see page 319 for the optimal value function and the barrier level in this model).

\section{Properties of Shareholder Risk MeAsures}

We want to establish some properties of the shareholder risk measure $(S R M)$ generated by the shareholder value function $v$ that is the solution of the optimization problem from the previous section, or more generally using a shareholder value function that is concave on $(0, \infty)$ and is linear and on $\left(x^{*}, \infty\right)$ with $x^{*} \geq 0$ (one can have $\overline{\overline{\bar{\nu}}}$ nind Figure 1 ). The objective is too see how different the SRM measures.

We recall that a convex risk measure $\rho: \mathcal{X} \rightarrow \mathbb{R}$ is defined by the following properties:

(1) Monotonicity: If $X \leq Y$, then $\rho(X) \geq \rho(Y)$. 
(2) Translation invariance (or cash invariance): If $m \in \mathbb{R}$, then $\rho(Y+m)=\rho(Y)-m$.

(3) Convexity: For any $\lambda \in(0,1), \rho(\lambda X+(1-\lambda) Y) \leq \lambda \rho(X)-(1-\lambda) \rho(Y)$.

4.1. Shareholder risk measures: a definition and some properties. Assume that we are time zero and an amount $k \in\left[0, x^{*}\right]$ of cash is available in the firm. Then, as described in the previous section, the shareholders value their shares as $v(k)$. If a random cash flow $X$ is about to affect the cash available in the firm, then the shareholders measure the risk of this random cash flow by its impact on their shareholder value.

We therefore define the following:

Definition 4.1. (1) We denote by $\mathcal{X}$ the class of random variables $X$ on $(\Omega, \mathcal{F})$ such that $\mathbf{E}[v(k+X)]<\infty$. The (instantaneous) shareholder risk measure for any $X \in \mathcal{X}$ is defined as:

$$
S R M(X):=v(k)-\mathbf{E}[v(k+X)] .
$$

(2) We denote $\mathcal{X}_{k}:=\{X \in \mathcal{X} \mid X>-k\}$ i.e., the set of risks bounded below by $-k$. $\mathcal{X}_{k}$ is called the set of sustainable risks. Each risk in this class does not trigger the firm's default, since the firm has sufficient cash to absorb its possible losses.

(3) The set of random variables $X$ satisfying $\mathbf{E}[v(k+X)] \geq v(k)$ or equivalently $S R M(X) \leq$ 0 is called the set of acceptable risks.

The intuition behind the definitions above is simple. If the cash process of the firm is impacted by a random variable $X$, then the value of the cash process changes from $k$ to $k+X$ and the value function from $v(k)$ to $v(k+X)$. The SRM evaluates the impact on the value function just before the random shock arrives, i.e., when there is no time to adjust the dividend policy before the occurrence of the shock. If the risk $X$ is such that $\mathbf{E}[v(k+X)]<v(k)$ it would be more convenient for shareholders to refuse being exposed to the risk $X$. We say that such risks are not acceptable. Note that $\bar{\equiv}_{\text {cept }}$ of acceptability here is different in its interpretation from the one introduced in Delbaen and al. [3], where a risk is not acceptable when it requires additional capital. In our setting, shareholders take only the dec $\overline{\bar{\nu}}$ n of the dividend policy and we do not allow for new capital injections.

Notation 4.2. With any random variable $X \in \mathcal{X}$ we attach a random variable $X_{k} \in \mathcal{X}_{k}$ by:

$$
X_{k}:=X \mathbf{1}_{\{X>-k\}}-k \mathbf{1}_{\{X \leq-k\}}=\max \{X,-k\} .
$$

Proposition 4.3 (Basic properties of the shareholder risk measure). SRM satisfies the following properties:

(i) $\operatorname{SRM}(0)=0$. More generally, if $X$ is such that $X_{k}=0$ a.s., then $\operatorname{SRM}(X)=0$.

(ii) General upper bound: $\operatorname{SRM}(X) \leq v(k)$.

(iii) Monotonicity: if $X$ and $Y$ are such that $X_{k}>Y_{k}$ a.s. then $S R M(X)<S R M(Y)$.

(iv) For $X \geq x^{*}-k$ and $m \geq x^{*}-(k+X)$ SRM has the property of cash invariance:

$$
S R M(X+m)=S R M(X)-m \text {. }
$$

(v) Internality (partial): if $X$ and $Y$ are such that $X_{k} \geq Y_{k}$ a.s., then for $\lambda \in(0,1)$, $\min \{S R M(X), S R M(Y)\} \leq S R M(\lambda X+(1-\lambda) Y) \leq \max \{S R M(X), S R M(Y)\}$.

(vi) Law equivariance: if $X$ and $Y$ are such that $X_{k}$ and $Y_{k}$ have the same distribution, then $\operatorname{SRM}(X)=\operatorname{SRM}(Y)$. 
Proof. (iii) Since $X_{k}>Y_{k}$ a.s. and $v$ is strictly increasing on $[0, \infty)$, we have that $v(k+$ $X)=v\left(k+X_{k}\right)>v\left(k+Y_{k}\right)=v(k+Y)$ and the result follows from the definition of SRM.

(iv) consequence of the fact that $v(x)$ is linear for $x>x^{*}$.

(v) We suppose that $X$ and $Y$ are such that $X_{k} \geq Y_{k}$ a.s.. In this case $v(k+X) \geq v(k+Y)$ a.s. and therefore:

$$
\begin{aligned}
\mathbf{E}[v(k+\lambda X+(1-\lambda) Y)] & \geq \mathbf{E}[\min (v(k+X), v(k+Y))] \\
& =\mathbf{E}[v(k+Y))]=\min \{\mathbf{E}[v(k+X)], \mathbf{E}[v(k+Y)]\}
\end{aligned}
$$

and:

$$
\begin{aligned}
\mathbf{E}[v(k+\lambda X+(1-\lambda) Y)] & \leq \mathbf{E}[\max (v(k+X), v(k+Y))] \\
& =\mathbf{E}[v(k+X))]=\max \{\mathbf{E}[v(k+X)], \mathbf{E}[v(k+Y)]\}
\end{aligned}
$$

We now write an equivalent form for the acceptable $S R M$, which emphasizes some similarities and differences with a class of law-equivariant or law-determined risk measures (also known as law-invariant risk measures). Kusuoka [22] has shown that every law equivariant, comonotonically additive risk measure can be represented as the expectation of the risk under a convexly distorted distribution. Namely, there exists a distortion $D$ (i.e., $D:[0,1] \rightarrow[0,1]$ increasing, right continuous, with $D(0)=0$ and $D(1)=1$ ) which is convex such that:

$$
\rho(X)=-\int_{0}^{1} F_{X}^{-1}(u) d D(u)=-\int_{\mathbb{R}} x d D \circ F_{X}(x),
$$

where $F_{X}(x):=\mathbf{P}(X \leq x)$.

Notation 4.4. On the interval $\left[0, x^{*}\right]$, the function $v$ is strictly concave therefore $v^{\prime}$ is invertible. We denote by $\left(v^{\prime}\right)^{-1}$ the inverse of $v^{\prime}$. By the mean value theorem, we have that for $x>-k$, there exists a point $y$ between $k$ and $x+k$ such that $v(k+x)-v(k)=x v^{\prime}(y)$. Since we have $k \in\left(0, x^{*}\right)$, the point $y$ also satisfies $y \in\left(0, x^{*}\right)$. Therefore, we define the function:

$$
g_{k}(x)=: \begin{cases}k & \text { if } x=0, \\ \left(v^{\prime}\right)^{-1}\left(\frac{v(k+x)-v(k)}{x}\right) & \text { if } x \in \mathbb{R}^{*}\end{cases}
$$

We also introduce the notations:

$$
\begin{aligned}
F_{X}(x) & :=\mathbf{P}(X \leq x) \\
F_{X_{k}}(x) & :=\mathbf{P}\left(X_{k} \leq x\right)
\end{aligned}
$$

Proposition 4.5 (Representation of the shareholder risk measure).

$$
S R M(X)=-\int_{\mathbb{R}} x v^{\prime}\left(g_{k}(x)\right) d F_{X_{k}}(x)
$$

Therefore:

(i) If $X>0$ a.s., we have that $\int_{\mathbb{R}} x v^{\prime}\left(g_{k}(x)\right) d F_{X_{k}}(x) \geq \mathbf{E}\left(X_{k}\right)=\mathbf{E}[X]$ hence:

$$
\operatorname{SRM}(X) \leq-\mathbf{E}(X)
$$


(ii) If $X<0$ a.s., we have that $\int_{\mathbb{R}} x v^{\prime}\left(g_{k}(x)\right) d F_{X_{k}}(x) \leq \mathbf{E}\left[X_{k}\right]$ hence:

$$
\operatorname{SRM}(X) \geq-\mathbf{E}\left(X_{k}\right)
$$

Let us comment on the above representations of $\rho$ versus $S R M$. Any law invariant commonotone risk measure for $X$ can be written as an expected value of $-X$ under a new probability measure. This probability measure is obtained by distorting the law of $X$ using a given function $D$ (thus for risks having different laws we get different probabilities after distortion). When computing the $S R M$ for $X$, we also compute the expectation of $-X$, but under a measure which is not a probability measure anymore. More precisely, because $v^{\prime}(x) \geq 1$ on $\mathbb{R}_{+}$we have that the new measure has a total mass which exceeds 1 . The new measure depends not only on the law of $X$ (in fact on the law of $X_{k}$ ), but also on the level of cash in the firm $k$. The higher the cash level, the lower the total mass of our measure change.

\subsection{Further properties of SRM: the case of bounded risks.}

Proposition 4.6 (Convexity for sustainable risks). Let $X \in \mathcal{X}_{k}$ and $Y \in \mathcal{X}_{k}$. Then, for any $\lambda \in[0,1]$ we have:

$$
\operatorname{SRM}(\lambda X+(1-\lambda) Y) \leq \lambda S R M(X)+(1-\lambda) S R M(Y) .
$$

The same holds, if, more generally, $\{X<-k\}=\{Y<-k\}$ (thus $X$ and $Y$ needn't be sustainable).

Proof. $\mathcal{X}_{k}$ is convex and $v$ is concave on $\mathbb{R}_{+}$. Hence for $X \in \mathcal{X}_{k}$ and $Y \in \mathcal{X}_{k}$ :

$$
\begin{aligned}
S R M(\lambda X+(1-\lambda) Y) & =v(k)-\mathbf{E}[v(\lambda(k+X)+(1-\lambda)(k+Y))] \\
& \leq v(k)-\mathbf{E}[\lambda v(k+X)+(1-\lambda) v(k+Y)] \\
& =\lambda \operatorname{SRM}(X)+(1-\lambda) S R M(Y) .
\end{aligned}
$$

The same holds if $\{X<-k\}=\{Y<-k\}$, because for all $\lambda \in[0,1]$ we have $\{X<-k\}=$ $\{\lambda X+(1-\lambda) Y<-k\}=\{Y<-k\}$.

Proposition 4.7 (Translation by a constant). Suppose that $X \in\left[x^{\min }, x^{\max }\right]$ for $x^{\max }, x^{\min }$ constants and we take $m$ constant.

(1) For moderate values of $m$, namely: if $-v\left(k+x^{\min }\right) \leq m<0$ or $0<m \leq v^{-1}(m)-$ $\left(k+x^{\max }\right)$, then:

$$
\operatorname{SRM}(X+m) \geq S R M(X)-m
$$

(2) For extreme values of $m$, namely: if $m<-v\left(k+x^{\max }\right)$ or $m>\max \left(v^{-1}(m)-(k+\right.$ $\left.\left.x^{\min }\right) ; 0\right)$, then:

$$
\operatorname{SRM}(X+m) \leq S R M(X)-m
$$

Proof. Let us suppose that $m<0$.

If $k+X+m \geq 0$, then necessarily $k+X>0$ and $v(k+X)-v(k+X+m) \geq-m$ (because $v^{\prime}(x) \geq 1$ for $x \in \mathbb{R}_{+}$and we can use the mean value theorem). It follows that $S R M(X+m) \geq S R M(X)-m$.

If $k+X+m<0$, and $k+X \leq 0$ then $v(k+X)=v(k+X+m)=0$ and hence, $v(k+X)-v(k+X+m)=0<-m$. It follows in this case that $S R M(X+m)<S R M(X)-m$.

If $k+X+m<0$, and $k+X \in\left[0, v^{-1}(-m)\right]$ then $v(k+X)-v(k+X+m)=v(k+X)<-m$, therefore $\operatorname{SRM}(X+m)<S R M(X)-m$. 
If $k+X+m<0$, and $k+X \in\left[v^{-1}(-m),-m\right]$ then $v(k+X)-v(k+X+m)=v(k+X)>$ $-m$, therefore $\operatorname{SRM}(X+m)>S R M(X)-m$.

Summarizing the above we get: If $m<0$ constant, then:

- If $X \geq v^{-1}(-m)-k$, then $\operatorname{SRM}(X+m) \geq S R M(X)-m$;

- If $X \leq v^{-1}(-m)-k$, then $\operatorname{SRM}(X+m) \leq S R M(X)-m$.

We now suppose that $m>0$.

If $v(k+X+m) \leq m$, then necessarily $k+X<0$ and $v(k+X)-v(k+X+m)=$ $-v(k+X+m) \geq-m$. It follows that $\operatorname{SRM}(X+m) \geq S R M(X)-m$.

If $v(k+X+m)>m$, and $k+X \leq 0$ then $v(k+X)-v(k+X+m)=-v(k+X+m)<-m$. It follows in this case that $\operatorname{SRM}(X+m)<S R M(X)-m$.

If $v(k+X+m)>m$, and $k+X>0$ then we also have $v(k+X)-v(k+X+m)<-m$ (since $v^{\prime}(x) \geq 1$ for $x \in \mathbb{R}_{+}$and we can use the mean value theorem) hence $\operatorname{SRM}(X+m)<$ $S R M(X)-m$.

Summarizing for $m>0$, we get:

- If $X \geq v^{-1}(m)-m-k$, then $\operatorname{SRM}(X+m) \leq S R M(X)-m$;

- If $X \leq v^{-1}(m)-m-k$, then $\operatorname{SRM}(X+m) \geq S R M(X)-m$.

Combining the results for $m>0$ and $m<0$ we get the result stated in the proposition.

One can notice that the following result was proved inside the proof of Proposition 4.7 (where in a first step we were not using upper and lower bounds for $X$ ):

Corollary 4.8. Suppose that $X \in \mathcal{X}_{k}$ and $m$ constant such that $X+m \in \mathcal{X}_{k}$. Then, the following hold:

(1) If $m<0$ then $\operatorname{SRM}(X+m) \geq S R M(X)-m$;

(2) If $m>0$ then $\operatorname{SRM}(X+m) \leq S R M(X)-m$.

The corollary above emphasizes that SRM fails to be cash invariant even when restricted to the case of sustainable risks.

For convex risk measures, the property of cash invariance is also questionable, for instance in the context of uncertainty about the interest rates. El Karoui and Ravanelli [13] propose to replace it with a relaxed version, called cash subadditivity (see also Cerreia-Vioglio et al. [9]). In the context of capital requirements, the meaning of cash subadditivity is that, when $m$ dollars are subtracted from a future position, the present capital requirement cannot be increased by more that $m$ dollars. We should mention that there is a temporal aspect embedded in this reasoning (namely that the risk is to be materialized at a future date), so that $\equiv$ directly comparable to our framework, as we treat instantaneous risks. However, we can see here again that what is desirable from regulators' perspective, does not naturally fit the shareholder's preferences. From the viewpoint of SRM, when a position is decreased by $m$ dollars, the risk increases by more that $m$ dollars, we have thus cash superadditivity. Intuitively, cash has more value when kept inside the company (except in the region where dividend distributions occur, as characterized in Proposition 4.3 (iv)).

The set $\mathcal{X}_{k}$ of sustainable risks has the convenient property of being a convex set. Therefore, we can construct a convex risk measure for $\mathcal{X}_{k}$ as follows. Let us denote by

$$
\mathcal{S}_{k}:=\left\{X \in \mathcal{X}_{k} \mid \operatorname{SRM}(X) \geq 0\right\}
$$


4.3. Risk magnification. Another property that has been mentioned in the literature as a desirable property for risk measures is the so-called star shapedness (or more precisely, 0-star shapedness): when a risk is magnified by a factor greater that 1 , the risk measure increases more than proportionally (see Cerreia-Vioglio and al. [9]). This is a weakening of the property of positive homogeneity, as follows:

$$
\rho(\lambda X) \geq \lambda \rho(X) \text { for } \lambda \in[1, \infty) .
$$

Let us note that when the reverse inequality holds, the function is called $\infty$-star shaped.

We now analyze the behavior of SRM in case of risk magnification. Since our risk measures depend also on the cash level, the scaling of the risk $X$ needs to take place i a scaling of the level of the cash $k$, if one wants to ensure ect comparison. In other words, we need to think of $S R M$ as a two dimensional function. Therefore, we denote $S R M(X \mid k) \quad \times$ the risk measure for $X$ conditional to the cash level being $k$.

Proposition 4.10. (1) If $X \geq 0$ then

$$
S R M(\lambda X \mid \lambda k) \geq \lambda S R M(X \mid k) \text { for } \lambda \in[1, \infty),
$$

that is, when restricted to positive risks, SRM is 0-star shaped.

(2) If $X \leq 0$ then

$$
\operatorname{SRM}(\lambda X \mid \lambda k) \leq \lambda S R M(X \mid k) \text { for } \lambda \in[1, \infty),
$$

that is, when restricted to negative risks, SRM is $\infty$-star shaped. 
Proof. We take $\lambda \in[1, \infty)$ and denote $\psi(x):=v(\lambda x)-\lambda v(x)$. For $x \leq 0$ we have $\psi(x)=$ 0 ; for $x>0$ : $\psi^{\prime}(x)=\lambda\left(v^{\prime}(\lambda x)-v^{\prime}(x)\right) \leq 0$, i.e., $\psi$ is a negative, decreasing function (hence the function $v$ is $\infty$-star shaped). This ensure the stated properties of SRM, since: $S R M(\lambda X \mid \lambda k)=\lambda S R M(X \mid k)-\mathbf{E}[\psi(k+X)-\psi(k)]$

Here, the financial interpretation is also clear: consolidation leads to more acceptance of the negative shocks. On the contrary, in absence of negative shocks, distinct, smaller entities are to be preferred.

\section{Risk meAsures Associated With CASH FlOWS ARRIVING AT A FUTURE DATE}

Let us now assume that the random shock $X$ will affect the cash flow process at a future date $T \geq 0$, the risk is hence characterized by the couple $(T, X)$. We assume that the shareholders can implement dividend strategies optimally, anticipating the random shock $(T, X)$. In this case, the value function will be different from the one characterized in Section 3 and the definition of $S R M$ should be extended to reflect the anticipation of shocks that arrive in the future.

For simplicity, we only consider here the case where the risk $(T, X)$ has the characteristics:

(1) $T$ is a stopping time in the filtration $\left(\mathcal{F}_{t}\right)$ generated by the cash process $\left(C_{t}\right)$.

(2) $X$ is a random variable independent from $\mathcal{F}_{\infty}$.

This framework covers some typical situations: cash flows arriving at a fixed date, or at jumps of the Lévy process (the latter characterizes a situation where the perception d言he size of the shock suffers an adjustment but not the arrival rate). This framework could be extended, but we want to keep the discussion simple.

We now define the cash process in presence of the random shock $(T, X)$ and a dividend process $\left(\xi_{t}\right)$, as a stochastic process $\left(\tilde{C}_{t}\right)$ with dynamics:

$$
\begin{aligned}
& d \tilde{C}_{t}=d C_{t}+X d \mathbf{1}_{\{T \leq t\}} \quad \text { for } t \in[0, \infty) \\
& \tilde{C}_{0^{-}}=k
\end{aligned}
$$

The dependence in the chosen dividend strategy $\xi$ is implicit via the process $C$.

We denote by $\left(\mathcal{G}_{t}\right)$ the natural augmented filtration of the process $\left(\tilde{C}_{t}\right)$. We have that $\mathcal{G}_{t}=\mathcal{F}_{t} \vee \sigma\left(X \mathbf{1}_{\{T \leq s\}}, s \leq t\right)=\mathcal{F}_{t} \vee \sigma\left(X \mathbf{1}_{\{T \leq t\}}\right)$ (the last equality holds because $T$ is considered a stopping time in the filtration $\left.\left(\mathcal{F}_{t}\right)\right)$. In particular $\mathcal{F}_{t}=\mathcal{G}_{t}$ for $t<T$. Let:

$$
\tilde{\tau}(t):=\inf \left\{s \geq t \mid \tilde{C}_{s}<0\right\}, \quad t \geq 0
$$

i.e., $\tilde{\tau}(t)$ is the first time after time $t$ when the cash process is negative, in presence of the random shock $(T, X)$. Similarily, we define for the process $\left(C_{t}\right)$, i.e., in absence of the random shock $(T, X)$ :

$$
\tau(t):=\inf \left\{s \geq t \mid C_{s}<0\right\}, \quad t \geq 0 .
$$

For simplicity, we write $\tilde{\tau}$ (respectively $\tau$ ) for $\tilde{\tau}(0)$ (respectively $\tau(0)$ ). The optimal dividend strategy after time $t$ is given as a solution of the problem:

$$
V_{t}:=\sup _{\xi \in \tilde{\mathcal{A}}_{t}} \mathbf{E}\left[\int_{[t, \tilde{\tau}(t)]} e^{-\rho(s-t)} d \xi_{s} \mid \mathcal{G}_{t}\right],
$$

with $\tilde{\mathcal{A}}_{t}=\tilde{\mathcal{A}}\left(t, \tilde{C}_{t}\right)$, where $\tilde{\mathcal{A}}(t, x)$ is the set of admissible controls after time $t$, that is, the set of $\left(\mathcal{G}_{t}\right)$-adapted, nondecreasing, càdlàg processes $\xi$ such that a unique strong solution 
$\left(\tilde{C}_{s}\right)_{s \geq t}$ exists conditionally on $\left\{\tilde{C}_{t^{-}}=x\right\}$, and such that for any stopping times $t \leq S \leq T$ we have: $\int_{S}^{T} \tilde{C}_{s} d \xi_{s} \geq 0$. This last condition ensures that no dividends are pay $\overline{\overline{\bar{v}}}$ hat would exceed the cash reserve available. We shall consider $\left(V_{t}\right)$ to be right-continuous.

For defining the dynamic version of the SRM, we need to write more precisely the value function before the random shock occurs. For this purpose, we introduce the two dimensional process $Z=\left(Z^{1}, Z^{2}\right), \mathbb{R}_{+} \times \mathbb{R}$-valued with components having the dynamics: $d Z_{s}^{1}=d s$, $d Z_{s}^{2}=d C_{Z_{0}^{1}+s}, s \geq 0$. We introduce a family of probability measures $\left(\mathbf{P}^{(t, x)}\right)_{\left(t \in \mathbb{R}_{+}, x \in \mathbb{R}\right)}$ on $\left(\mathbb{R}_{+} \times \mathbb{R} \times \Omega, \mathcal{B}\left(\mathbb{R}_{+} \times \mathbb{R}\right) \otimes \mathcal{F}\right)$ such that under $\mathbf{P}^{(t, x)}$, the process $Z$ starts at $(t, x)$ (i.e., $\left.Z_{0}^{1}=t, Z_{0}^{2}=x\right)$ ). We denote hereafter $\mathbf{E}^{(t, x)}$ the expectation under $\mathbf{P}^{(t, x)}$.

We also define:

$$
\tilde{v}(t, x):=\sup _{\xi \in \tilde{\mathcal{A}}(t, x)} \mathbf{E}^{(t, x)}\left[\int_{[0, \tilde{\tau}]} e^{-\rho s} d \xi_{s}\right] .
$$

By (slight) abuse of notation we have used above $\tilde{\tau}=\inf \left\{s \geq 0 \mid Z_{s}^{2}<0\right\}$.

We now give an expression of the process $V$ using the functions $v$ and $\tilde{v}$.

Proposition 5.1. The process $V$ is given by:

$$
V_{t}= \begin{cases}\tilde{v}\left(t, C_{t}\right) & \text { if } t<T \\ v\left(\tilde{C}_{t}\right) & \text { if } t \geq T\end{cases}
$$

Proof. Recall that for all $t<T$ we have that $\tilde{C}_{t}=C_{t}$ and therefore $\mathcal{F}_{t}=\mathcal{G}_{t}$ and $\tilde{\mathcal{A}}_{t}=$ $\tilde{\mathcal{A}}\left(t, C_{t}\right)$. Then:

$$
\tilde{v}\left(t, C_{t}\right)=\sup _{\xi \in \tilde{\mathcal{A}}\left(t, C_{t}\right)} \mathbf{E}\left[\int_{t^{-}}^{\tilde{\tau}(t)} e^{-\rho(s-t)} d \xi_{s} \mid \mathcal{G}_{t}\right]=V_{t}
$$

since it corresponds precisely to the problem in (5.3).

Once the random shock occurs, the problem becomes time homogeneous. Consequently, for $t \geq T$ we have: $\tilde{\mathcal{A}}\left(t, \tilde{C}_{t}\right)=\mathcal{A}\left(\tilde{C}_{t}\right)$, i.e., given the current cash level the admissible strategies are the same as in (3.3). We have therefore for $t \geq T$ :

$$
\begin{aligned}
V_{t} & =\sup _{\xi \in \tilde{\mathcal{A}}_{t}} \mathbf{E}\left[\int_{t^{-}}^{\tilde{\tau}(t)} e^{-\rho(s-t)} d \xi_{s} \mid \mathcal{F}_{t} \vee \sigma(X)\right] \\
& =\sup _{\xi \in \tilde{\mathcal{A}}_{t}} \mathbf{E}^{\left(t, \tilde{C}_{t}\right)}\left[\int_{0^{-}}^{\tilde{\tau}} e^{-\rho s} d \xi_{s}\right] \\
& =\sup _{\xi \in \mathcal{A}\left(\tilde{C}_{t}\right)} \mathbf{E}^{\tilde{C}_{t}}\left[\int_{0^{-}}^{\tau} e^{-\rho s} d \xi_{s}\right]=v\left(\tilde{C}_{t}\right) .
\end{aligned}
$$

Definition 5.2. We denote by $\mathcal{X}(T)$ the class of random variables $X$ on $(\Omega, \mathcal{F})$ such that $\mathbf{E}\left[v\left(C_{T}+X\right)\right]<\infty$ and where $T$ is an $\left(\mathcal{F}_{t}\right)$-stopping time. For any $X \in \mathcal{X}(T)$ and $t \leq T$, we define the time $t$ shareholder risk measure as the $\mathcal{F}_{t}$-measurable random variable:

$$
S R M_{t}(X):=\left(v\left(C_{t}\right)-\tilde{v}\left(t, C_{t}\right)\right) \mathbf{1}_{\{\tau>t\}} .
$$


The time $t$ shareholder risk measure, conditional on the cash level being $k \geq 0$ is denoted:

$$
S R M_{t}(X \mid k):=v(k)-\tilde{v}(t, k) .
$$

The $S R M_{t}(X)$ represents the impact on the shareholder value of the exogenous shock $(T, X)$, including the optimal readjustment of the dividend strategy from time $t$ on.

The next lemma is helpful in understanding the link between the instantaneous SRM and the dynamic counterpart taken at time $T$.

\section{Lemma 5.3.}

$$
\tilde{v}\left(T, C_{T}\right)=\mathbf{E}\left[v\left(C_{T}+X\right) \mid \mathcal{G}_{T-}\right]
$$

and:

$$
\tilde{v}(T, x)=\mathbf{E}[v(x+X)] .
$$

Proof. We have by definition of the function $\tilde{v}$ that:

$$
\tilde{v}\left(T, C_{T}\right)=\mathbf{E}\left[V_{T} \mid \mathcal{F}_{T}\right]=\mathbf{E}\left[v\left(\tilde{C}_{T}\right) \mid \mathcal{F}_{T}\right]
$$

Since $X$ is independent from the process $\left(C_{t}\right)$, hence from $\mathcal{F}_{T}$, we have

$$
\tilde{v}(T, x)=\mathbf{E}[v(x+X)] .
$$

To conclude, we need to show $\mathcal{F}_{T}=\mathcal{G}_{T-}$. Let us recall a less standard definition of $\mathcal{G}_{T-}$ which is:

$$
\mathcal{G}_{T-}=\sigma\left\{Y_{T},\left(Y_{t}\right) \text { any }\left(\mathcal{G}_{t}\right) \text {-adapted, left continuous process }\right\}
$$

(this can be obtained similarly to Ex. 4.20 p. 47 in [29]). By construction of the filtration $\left(\mathcal{G}_{t}\right)$, the class of $\left(\mathcal{F}_{t}\right)$ adapted left continuous processes stopped at $T$ is the same as the class of $\left(\mathcal{G}_{t}\right)$ adapted left continuous processes stopped at $T$, that is, $\mathcal{G}_{T-}=\mathcal{F}_{T-}$. Finally, the filtration $\left(\mathcal{F}_{t}\right)$ is generated by the cash process $\left(C_{t}\right)$ (which has no jumps at predictable times) hence is quasi left continuous, i.e. $\mathcal{F}_{T-}=\mathcal{F}_{T}$ for all stopping times $T$.

With the help of Lemma 5.3, we can make the link between the above definition and the instantaneous measure $S R M(X)$ that was introduced in the previous section:

(1) Conditionally on the cash level. We have:

$$
S R M_{T}(X \mid k)=(v(k)-E[v(k+X)])=S R M(X),
$$

that is, at the maturity $T$, the shareholder's risk measure coincideswith the instan- $\times$ taneous risk measure corresponding to the same cash level.

(2) Unconditionally on the cash level. In general, the instantaneous risk measure for $(T, X)$ is an $\mathcal{F}_{T}$-measurable random variable that can be written:

$$
S R M_{T}(X)=v\left(C_{T}\right)-\mathbf{E}\left[v\left(C_{T}+X\right) \mid \mathcal{G}_{T-}\right] .
$$

The process $\left(S R M_{t}(X), t \in[0, T]\right)$ is an $\left(\mathcal{F}_{t}\right)$ adapted process since it is a function of the cash process $C$ and time. Also, it is absorbed at 0 at the bankruptcy time $\tau$.

In order to further analyze the process $\left(S R M_{t}\right)$ it is necessary to specify the dynamics of the process $\left(C_{t}\right)$ in particular the implemented dividend strategy $\xi$. Let us now consider $\left(C_{t}\right)$ is the optimal cash process in absence of the random shock. In other words, we assume the implemented dividend strategy is the local time at $x^{*}$, i.e., the optimal barrier as in Section 3. In this case, we obtain the following result: 
Theorem 5.4. The discounted process $\left(e^{-\rho t} S R M_{t}(X)\right)_{t \in[0, T \wedge \tilde{\tau}]}$ is a submartingale.

Proof. Let us denote by $\left(\widetilde{L}_{t}^{*}\right)$ the local time at $x^{*}$ of the process $\tilde{C}$ and by $\left(L_{t}^{*}\right)$ is the local time at $x^{*}$ of the process $C$. Let us consider the dividend strategy that consists in paying a dividend process $\widetilde{L}^{*}$. This being one possible choice among the admissible dividend strategies in presence of the random shock $(T, X)$, and not necessarily optimal, we obtain that for $t<T \wedge \tau$ :

$$
\begin{aligned}
V_{t} & =\tilde{v}\left(t, C_{t}\right) \geq \mathbf{E}\left[\int_{[t, \widetilde{\tau}(t)]} e^{-\rho(s-t)} d \widetilde{L}_{s}^{*} \mid \mathcal{F}_{t}\right] \\
& =\mathbf{E}\left[\int_{[t, \widetilde{\tau}(t) \wedge T)} e^{-\rho(s-t)} d \widetilde{L}_{s}^{*}+\mathbf{1}_{\{\widetilde{\tau}(t) \geq T\}} e^{-\rho(T-t)} v\left(\widetilde{C}_{T}\right) \mid \mathcal{F}_{t}\right] \\
& =\mathbf{E}\left[\int_{[t, \tau(t) \wedge T)} e^{-\rho(s-t)} d L_{s}^{*}+\mathbf{1}_{\{\tau \geq T\}} e^{-\rho(T-t)} v\left(C_{T}+X\right) \mid \mathcal{F}_{t}\right] \\
& =v\left(C_{t}\right)+\mathbf{E}\left[\mathbf{1}_{\{\tau \geq T\}} e^{-\rho(T-t)}\left(v\left(C_{T}+X\right)-v\left(C_{T}\right)\right) \mid \mathcal{F}_{t}\right] .
\end{aligned}
$$

We have used the fact that on $[0, T) \tilde{C} \equiv C$ and $\tilde{C}_{T}=C_{T}+X$. Notice that even though the random variables $\tau(t)$ and $\tilde{\tau}(t)$ differ, the two sets $\{\tilde{\tau}(t) \geq T\}$ and $\{\tau(t) \geq T\}$ are equal, their complements being equal. Thus, we preferred to split the dividend flow in such a way that we can use this property.

It follows that, for $t \in[0, T]$ :

$$
S R M_{t}(X) \leq \mathbf{E}\left[e^{-\rho(T-t)} S R M_{T}(X) \mid \mathcal{F}_{t}\right]
$$

Using similar arguments we can show that for for $0 \leq s \leq t \leq T$ we have $S R M_{s}(X) \leq$ $\mathbf{E}\left[e^{-\rho(t-s)} S R M_{t}(X) \mid \mathcal{F}_{t}\right]$, which proves the statement.

The theorem can be interpreted as follows: the discounted risk measure increases itvarage when the time to the shock decreases. This is a consequence of the fact that when the time to the shock decreases, the shareholders have less time to adjust their dividend strategy before the random shock occurs, hence the impact on their value function will be higher.

Some additional inequalities can be easily derived from the Theorem 5.4 and Proposition 4.5, for risks that do not involve losses, that is $X \geq 0$ a.s.. However, it is not possible to establish simple and general relations when losses are involved (that is $\mathbf{P}(X<0)>0)$.

Corollary 5.5. If $X \geq 0$ a.s. and for any $s \leq t \leq T$, the following hold:

and therefore

$$
S R M_{s}(X) \leq-e^{-\rho(T-s)} \mathbf{E}\left[X \mid \mathcal{F}_{s}\right]
$$

$$
S R M_{s}\left(S R M_{t}(X)\right) \leq-S R M_{s}(X) .
$$

To conclude, we can remark under the assumed framework, the dynamic version of $S R M$ is not a time consistent risk measure in the way dynamic convex risk measures are (see for instance [1]).

\section{REFERENCES}

[1] Acciaio B. And I. Penner: Dynamic convex risk measures, Advanced Mathematical Methods for Finance, G. Di Nunno and B. Øksendal (eds.) Springer-Verlag Berlin Heidelberg, 1-34 (2011). 
[2] Albrecher H. and S. Thonhauser: Optimal dividend strategies for a risk process under force of interest. Insurance: Math. Econom., 43, 134-149 (2008).

[3] Artzner P., F. Delbaen, J. M. Eber and D. Heath: Coherent measures of risk, Math. Finance, 9, 203-228 (1999).

[4] Asmussen, S. And M. TAKsar: Controlled diffusion models for optimal dividend pay-out, Insurance: Math. Econom. 20, 1-15 (1997).

[5] Avram, F., Z. Palmowski, and M. R. Pistorius: On the optimal dividend problem for a spectrally negative Lévy process. Ann. Appl. Probab., 17(1),156-180 (2007).

[6] Azcue, P. And N. Muler: Optimal reinsurance and dividend distribution policies in the CramérLundberg model, Math. Finance, 15(2), 261-308 (2005).

[7] BANK, P. AND F. RiEdel: Optimal Consumption Choice under Uncertainty with Intertemporal Substitution, Ann. Appl. Probab., 11, 750-788 (2001).

[8] Belhaj, M.: Optimal Dividend Payments when Cash Reserves Follow a Jump-Diffusion Process, Math. Finance, 20(2), 313-325 (2010).

[9] Cerreia-Vioglio, S., F. Maccheroni, M. Marinacci and L. Montrucchio: Risk Measures: Rationality and Diversification, Math. Finance, 21, 743-774 (2011).

[10] Chaleyat-Maurel, M., N. El Karoui and B. Marchal: Réflexion discontinue et systèmes stochastiques, Ann. Probab., 8, 1049-1067 (1980).

[11] DE Finetti, B.: Su unimpostazion alternativa dell teoria collecttiva del rischio. In Trans. XVth Internat. Congress Actuaries, 2, 433-443 (1957).

[12] Eisenberg, J. And H. Schmidli: Optimal Control of Capital Injections by Reinsurance in a Diffusion Approximation, Bläter DGVM 30, 1-13 (2009).

[13] El Karoui, N. and C. Ravanelli: Cash Subadditive Risk Measures and Interest Rate Ambiguity, Math. Finance, 19(4), 561-590 (2009).

[14] Föllmer H. ANd A. Schied: Convex measures of risk and trading constraints. Finance Stochast., 6 , 429-447 (2002).

[15] Föllmer H. And A. Schied: Stochastic Finance: An Introduction in Discrete Time, Walter de Gruyter (2004).

[16] Frittelli M. and E. Rosazza Gianin: Putting order in risk measures, J. Banking Finance, 26, 1473-1486 (2002).

[17] Gerber H. U. Entscheidungskriterien fuer den zusammengesetzten Poissonprozess. Bulletin of the Swiss Association of Actuaries, 1-47 (1969).

[18] Gerber, H. U. And Shiu, E. S. W.: Optimal dividends: analysis with Brownian motion, N. Am. Actuar. J., 8(1):120 (2004).

[19] Jeanblanc-Picqué, M. And A. N. Shiryaev: Optimization of the flow of dividends. Russian Mathematical Surveys, 50(2): 257-277 (1995).

[20] Kyprianou, A. E. and Palmowski, Z.: Distributional study of de Finettis dividend problem for a general Lévy insurance risk process. J. Appl. Probab., 44(2):428-443 (2007).

[21] Kulenko, N. And H. Schmidli: Optimal Dividend Strategies in a Cramér Lundberg Model with Capital Injections, Insurance: Math. Econom.,43, pp. 270-278 (2008).

[22] Kusuoka S. On law-invariant coherent risk measures, Advances in Mathematical Economics 3, 83-95 (2001).

[23] Loeffen, R. L.: On optimality of the barrier strategy in de Finetti's dividend problem for spectral negative Lévy processes, The Annals of Applied Probability, 18(5), 1669-1680 (2008).

[24] von-Neumann, J., And O. Morgenstern: Theory of Games and Economic Behavior. Princeton: Princeton University Press (1947).

[25] J.-C. Rochet and S. Villeneuve: Corporate Portfolio Management, Annals of Finance, 1(3), 225243.

[26] ØKsendal, B. And A. Sulem: Applied Stochastic Control of Jump-Diffusions, Springer Verlag, second edition (2007).

[27] Schmidui, H.: Stochastic Control in Insurance, Springer Verlag, London (2008).

[28] Shreve, S.E., J.P. Lehoczky and D.P. Gaver: Optimal Consumption for General Diffusions with Reflecting and Absorbing Barriers, SIAM, J. Control and Optimization, 22(1), 55-75 (1984). 
1

2

3

4

5

6

7

8

9

10

11

12

13

14

15

16

17

18

19

20

21

22

23

24

25

26

27

28

29

30

31

32

33

34

35

36

37

38

39

40

41

42

43

44

45

46

47

48

49

50

51

52

53

54

55

56

57

58

59

60

[29] Revuz, D. And M. Yor: Continuous Martingales and Brownian Motion, Third edition, Springer, 1999. 


\section{SHAREHOLDER RISK MEASURES} ANSWER TO THE REVIEWER 1

We would like to thank the referee for a very careful reading and the many pertinent comments that have improved our paper, now that we have incorporated all of them.

We detail below our answers to the major points raised:

(1) (Here we simultaneously address point (4), which is of similar nature.) Following your suggestion we explain in more detail that, although the traditional approach to risk measures is normative, we adopt here a positive viewpoint: how private companies measure risks in the interest of their shareholders.

(2) Page 5, paragraph 5: we added this paragraph where we make more precise our point of view. This important point was missing. Of course, other approaches are possible, and could be considered in further research, as the ones you suggest. We choose rather to set our model in the framework of complete markets.

(3) Thank you for raising this point. A paragraph was added (penultimate paragraph, page 16).

(4) See (1).

(5) A subsection was added on star shapedness: 4.3., page 17, thank you for suggesting to look to this property.

(6)-(7)-(8) Done as suggested.

We have also carefully incorporated all the comments which were classified by the reviewer as minor. Additionally, we would like to draw the attention on the fact that a new paragraph on time consistency was added (last paragraph page 21) in response of a question of reviewer 2 .

Date: October 13, 2015. 
We would like to thank the referee for a very careful reading and the many pertinent comments that have improved our paper, now that we have incorporated all of them. We detail below our answers to the main points raised:

(1) It is common in the literature of singular control to consider the control process (the dividend process $\xi$ in our case) to be right continuous (see the book by Øksendal and Sulem for instance). The decision should not foresee future shocks; this property is insured by the fact that the control process is adapted. Choosing RC controls implies that the decision is not necessarily predictable. This feature does not have any unpleasant implications and has some advantages. Indeed, let us consider a predictable control $\xi$. Then, the typical situation where this would play a different role, is at jump times of the Lévy process. When $\xi$ needs to be increased just after such a jump, $\xi$ will be left continuous. Consequently, the controlled cash reserves will be neither left continuous nor right continuous at that jump. By replacing $\xi$ by $\xi_{-}$as the control process, we preserve RC sample path for the cash process. And the obtained cash process is indistinguishable from the first one. Therefore, one can without harm of have RC dividend processes.

(2) In fact, the paper mentions one example with jumps, see subsection 3.2.3.

(3) We care about emphasizing the limited liability for shareholders and the property $v(x)=0$ on $x \in(-\infty, 0)$ is perfectly reflecting this feature. Changing it as you suggests would lead to "infinite punishment" in case of default. In fact limited liability makes shareholders indifferent at default and they accept default as a downside of some risky positions. Our point is that diversification is not working in the usual way since shareholders are hedged in case of default. This feature would not be captured in the framework you suggest.

(4) Thank you for pointing our the paper by Bank and Riedel, which appears now in the bibliography. We decided to be more precise on our model specification (see paragraph 5 on page 5). Introducing utility functions as you suggest is an interesting topic that would require some deep investigation of the resulting value function and related risk measures. Also, dividends are money, hence their value is transferable in time for later consumption, hence we believe that our framework is coherent (the situation would be different in a non financial framework with consumption in the form of perishable goods for instance).

(5) The existence of a regular solution in the general case is not guaranteed, it depends on the Lévy measure under consideration. For instance, in the case of spectrally 
negative Lévy processes, some smoothness conditions of the $q$-scale function of the Lévy process have been shown to be sufficient conditions (see Loeffen [1] for more details).

We now answer the points raised by the reviewer in the section Remarks.

- This was justified in the point (3) above.

- The statement was removed.

- The assumptions are now to be found in front of Theorem 3.2 and we added two words on the financial motivations (but clearly the conditions are important for their mathematical implications).

- Yes, it was a typo, we corrected.

- Done.

- Done.

- This was justified in the point (3) above.

- This was justified in the point (3) above.

- Thank you, typos corrected.

- Yes, we agree that both assumptions are strong. We did not try the most general framework, but we were willing to give an idea of how the dynamic case would work. Assumption (1) can be replaced with the assumption that the arrival time is independent from the filtration of the process $C$ (the computations are feasible if we assume some distribution for the arrival time $T$, as for instance exponential). Then, together with assumption (2) it would characterize a situation of an independent risks (both in the arrival and the amount). Treating this case would require some additional projections on the filtration $\left(\mathcal{F}_{t}\right)$. This case is a bit more elaborate to treat and does not cover some typical situations firms are facing: risks arriving at a fixed maturity and risks that arrive at the jumps of the Lévy process (this is a situation when the perception on the size of the shock suffers a change but not the arrival rate).

- $d$ stands for the derivative.

- Time consistency: SRM is not time consistent. A paragraph was added (last paragraph page 21).

We would also like to draw the attention upon the introduction of a new subsection (4.3. page 17) in response to a question of Reviewer 1 . Also, the introduction was very slightly rewritten.

\section{REFERENCES}

[1] Loeffen, R. L.: On optimality of the barrier strategy in de Finetti's dividend problem for spectral negative Lévy processes, The Annals of Applied Probability, 18(5), 1669-1680 (2008). 